\title{
Norois
}

Environnement, aménagement, société

$210 \mid 2009 / 1$

L'agriculture et les filières agro-alimentaires en France et en Argentine

\section{Réflexions critiques pour penser le territoire argentin aujourd'hui. Le prétexte du soja}

Soya in Argentina, a way of thinking the present territory

La soja en Argentina. Piensar el territorio hoy

José María Cóccaro et Gabriela Inés Maldonado

\section{(2) OpenEdition}

Journals

\section{Édition électronique}

URL : https://journals.openedition.org/norois/2774

DOI : $10.4000 /$ norois. 2774

ISBN : 978-2-7535-1558-1

ISSN : 1760-8546

Éditeur

Presses universitaires de Rennes

Édition imprimée

Date de publication : 1 mars 2009

Pagination : 43-68

ISBN : 978-2-7535-0844-6

ISSN : 0029-182X

Référence électronique

José María Cóccaro et Gabriela Inés Maldonado, «Réflexions critiques pour penser le territoire argentin aujourd'hui. Le prétexte du soja », Norois [En ligne], 210 | 2009/1, mis en ligne le 01 mars 2011, consulté le 14 janvier 2022. URL : http://journals.openedition.org/norois/2774 ; DOI : https:// doi.org/10.4000/norois. 2774

Ce document a été généré automatiquement le 14 janvier 2022.

(ㄷ) Tous droits réservés 


\title{
Réflexions critiques pour penser le territoire argentin aujourd'hui. Le prétexte du soja
}

\author{
Soya in Argentina, a way of thinking the present territory \\ La soja en Argentina. Piensar el territorio hoy
}

José María Cóccaro et Gabriela Inés Maldonado

\section{NOTE DE L'ÉDITEUR}

Cet article a été reçu le 13 juin 2008 et définitivement accepté le 5 février 2009.

1 Cette proposition théorique, conceptuelle et méthodologique s'inscrit au sein d'une ligne de recherche qui a commencé à se dessiner aux cours de différents projets, ces dix dernières années. Parmi ces recherches, il y eut différentes analyses localisées, notamment dans le sud de la province de Córdoba ${ }^{1}$. L'accent a notamment été mis sur l'effet de l'incitation à la production de soja et l'émergence de problèmes environnementaux dans le Sur Cordobés ${ }^{2}$ (Cóccaro et al., 1998-2002 ; Valenzuela et al., 2003-2004; Sosa et al., 2005-2006, Valenzuela et al., 2007-2008, Maldonado, 2008). Ces travaux monographiques ont en commun d'avoir joué le rôle de "prétexte » dans l'interprétation du processus de production du territoire en Argentine.

2 Des géographes tels qu'Henri Lefebvre (1984), Milton Santos (1985, 1994, 1996, 2000), Guy Di Méo (1998), Doreen Massey (2005), María Laura Silveira (1999, 2001, 2003, 2008), David Harvey (1998, 2004, 2007), ont développé et construit une structure conceptuelle cohérente à partir d'une géographie critique, sur laquelle s'appuient nos traitements théoriques et méthodologiques de problématiques " localisées » (Maldonado et Cóccaro, 2008). L'espace géographique est ainsi lu en tant que processus de production $\mathrm{du}$ territoire (comme état de situation de ce processus de production) et de l'environnement (comme évaluation de la qualité de celui-ci). Cette seconde approche 
s'est accentuée au cours de nos recherches. Par conséquent, l'espace-temporalité est la catégorie d'analyse pertinente. La géographie dans son ensemble devient donc géographie historique. Dans cette perspective, il est essentiel d'examiner le climat d'idées qui a alimenté la construction d'une hiérarchie de valeurs, de savoirs et de comportements fonctionnels, aux différentes étapes historiques du capitalisme. Ceci est particulièrement évident pendant la période néolibérale et conservatrice qui a caractérisé l'Argentine pendant les dix dernières années $\mathrm{du} \mathrm{xx}^{\mathrm{e}}$ siècle.

3 L'ambition de cet article, à partir de la question « prétexte » du soja, est de proposer des façons de conceptualiser l'espace, pour un traitement éthique et politique des problèmes du territoire, dans une perspective humaniste de responsabilité sociale et de contribution au bien public.

\section{Le territoire, une manière d' « être » et de « faire »}

4 Le territoire argentin a été, notamment à partir de la deuxième moitié du xix siècle, le témoignage visible des adaptations tardives aux contradictions propres à l'accumulation du capital. L'histoire de l'Argentine est étroitement liée à l'exploitation de ses « ressources naturelles » en général, et plus particulièrement à la production agricole. Le modèle agro-exportateur (1880-1930) a été la structure économique qui a défini la structuration et la consolidation du pays à cette époque. La consolidation de l'étape agro-exportatrice a exigé l'appropriation par la force, des terres appartenant à des peuples originaires ${ }^{3}$. "La conquête a permis l'appropriation privée et intégrale de la terre pampéenne et a consolidé le caractère et le modèle d'appropriation du grand propriétaire foncier, source de l'accumulation initiale de la classe dominante locale ${ }^{4}$ ” (Rapoport, 2007, p. 7). C'est ainsi que le marché foncier spéculatif a incorporé des terres qui ont été à l'origine du régime de " arrendamiento ${ }^{5}$ » et de " aparcería ${ }^{6}$ », principale cause historique de la surexploitation et de la quête de revenus à court terme. On appliquait ainsi, bien que tardivement, la " théorie des avantages comparatifs " énoncée par David Ricardo au XvIII ${ }^{e}$ siècle, fonctionnels à la théorie de l'expansion du capital. L'Argentine s'incorporait au marché mondial à travers le revenu différentiel généré par la capacité productive naturelle du sol.

5 À partir de cette phase fondatrice, l'activité agricole a certes souffert de crises régulières, fonctionnelles, nécessaires aux nouveaux processus d'accumulation du capital. Mais ce modèle culturel, enraciné, construit et soutenu par le débordement du revenu agricole, n'avait pas l'intention de s'orienter vers une diversification du système productif. C'est pourquoi, le secteur agricole s'établit encore de nos jours, comme fondateur des bases pour l'État moderne et se sent donc le « maître du pays ». En effet, les matérialisations de capital fixe de la période agro-exportatrice, tel que le réseau ferroviaire, persistent encore. Ce modèle, auquel nous avons donné le nom de fondateur (1880-1930), a déployé un réseau ferroviaire qui permettait la communication, à cette époque, entre la principale région productive exportatrice et le reste du monde. La figure 1 représente les entités écologiques du territoire argentin. On peut y observer la haute densité de chemins de fer, construits principalement entre 1880 et 1914, qui traversent les biomes de Pastizal Pampeano ${ }^{7}$ et d'Espinal ${ }^{8}$. Aujourd'hui, ces biomes sont d'insaisissables héritages face à l'avancée ravageuse de la frontière agricole pampéenne. Par ailleurs, la quasi-totalité du système ferroviaire a été mis hors service pendant les années 1990. Cette image territoriale constitue un point de départ essentiel 
pour comprendre, dans le processus historique de production du territoire, l'impact de la continuité (avec certaines ruptures) de la " reprimarisation" " de l'économie argentine. Cette tendance commence avec le modèle agro-exportateur. Elle est reprise avec un nouvel élan, pendant la période d'ouverture économique et d'endettement externe, lors de la dictature civile et militaire (1976-1982). Elle est approfondie au cours de la période néolibérale conservatrice des années 1990, avec la déréglementation et la réforme de l'État.

Le processus d' « agriculturisation ${ }^{10}$ » commencé dans les années 1970, prend de l'élan au cours des quinze dernières années, avec un rôle largement dominant du soja transgénique dans le contexte actuel d'inflation mondiale produite par la demande d'aliments et de biocarburants. La sojisation ${ }^{11}$ est la matérialisation d'une manière de penser le territoire aujourd'hui. C'est le reflet du poids de l'héritage de la grande expansion productive de la région pampéenne argentine, initiée au cours de la deuxième moitié $\mathrm{du} \mathrm{xIx}^{\mathrm{e}}$ siècle, lors de la période dite agro-exportatrice. C'est le témoignage présent de l'histoire d'un territoire, organisé autour d'une région centrale (la Pampa) à forte productivité agricole, entourée d'économies régionales, orientées principalement vers la consommation intérieure. C'est l'instrument actuel d'invasion et de conquête des régions extra-pampéennes. C'est l'image de la " pampéanisation ${ }^{12}$ » du territoire. C'est l'or vert qui unifie le vieux et le nouveau « campo ${ }^{13}$ ». C'est le cheval de Troie ${ }^{14}$ de l'appropriation du revenu agricole au moyen des espaces nécessaires à l'expansion du capital.

7 Aller d'aujourd'hui à hier, d'hier à aujourd'hui, telles sont les fenêtres nécessaires pour observer les transformations profondes du territoire argentin comme le résultat dialectique des relations entre les structures de pouvoir et de décision, relatives à la géographie historique de l'accumulation capitaliste. De là, notre conception dialectique de l'espace - dans le contexte d'un processus de production - en tant que produit et producteur de la société et notre conception du territoire en tant qu'état situationnel de ce processus dialectique de production. Le territoire est un objet de travail. C'est un moyen de travail et, en tant que tel, un moyen de production. C'est aussi bien le contenant que le contenu des conditions matérielles pour le processus de travail. Il fait partie de la production de la société. Il exprime des manières d' « être » et de « faire ». 
Figure 1 : Biomes et réseau ferroviaire argentins Eco regions and railway network in Argentina

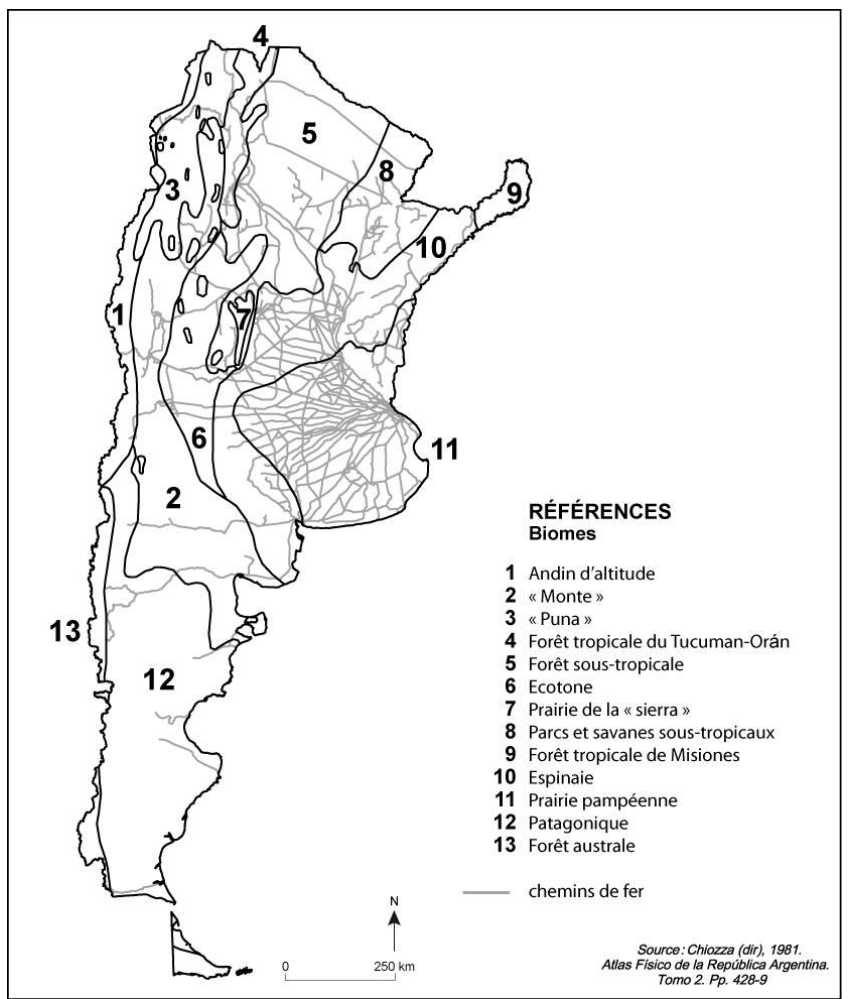

\section{Le territoire et le revenu agricole}

De nos jours, le débat sur la question agricole tourne autour de la concentration de la propriété territoriale, la concentration des capitaux dans la production, le processus d'agriculturisation et de sojisation, et les conséquences sociales et environnementales de l'expansion du capitalisme dans le domaine agricole. C'est sur ces axes que s'appuie une brève analyse contextuelle sur l'état de situation actuelle du secteur agricole argentin en général. La production d'un revenu agricole sans précédent et le débat sur son origine et son appropriation, est à la base de l'explication des processus en cours. Le fait que la terre soit limitée, qu'elle puisse être appropriée et qu'elle soit hétérogène (en fonction de sa qualité et de sa situation géographique) permet à son possesseur de bénéficier de revenus. Dans le présent article, nous ne visons pas à approfondir ce sujet mais à délimiter sa portée conceptuelle afin d'aborder la question de penser le territoire.

\section{Le revenu foncier}

Le revenu foncier a été défini par K. Marx comme la somme de deux types de revenu : le revenu absolu et le revenu différentiel quel qu'il soit. Le revenu absolu résulte de la différente composition organique du capital de l'activité agricole, par rapport à l'activité industrielle (en agriculture, le capital variable est supérieur au capital constant). Ce revenu est le résultat des caractéristiques de la production agricole, par rapport aux autres activités productives. Par contre, le revenu différentiel est le résultat des divers coûts de production et de productivité dans le domaine de l'activité 
agricole. Dans le premier cas, la différence est marquée par le degré de fertilité du sol et par la localisation des terres. Mais dans le second cas, la différence résulte des investissements des capitaux successifs sur la même terre. De cette manière, des terres ayant des degrés de productivité hétérogènes rentrent dans la production, mais le prix du marché est déterminé par les produits provenant des terres qui ont le coût de production le plus élevé (celles qui ne produisent que le revenu absolu). C'est ainsi qu'une terre ayant une productivité plus importante que la précédente, génère un gain extraordinaire qui, perçu par la propriété territoriale, devient revenu différentiel. Ce dernier est fréquemment plus élevé que le revenu absolu. La source du revenu foncier est la plus-value. Même si la force de la nature est la première responsable de l'accroissement de la productivité de la terre, c'est le travail qui est le seul capable de lui attribuer une valeur. Le revenu (plus-value dans toutes ses formes) est le tout ou la partie du gain extraordinaire qui, au lieu d'être saisi par le capitaliste, est intercepté par la propriété territoriale, en d'autres termes le propriétaire foncier.

10 Dans cet article, nous abordons la discussion sur le revenu car il est à la base de l'explication des processus de concentration de la propriété et de capital dans la production, à travers l'agriculturisation et la sojisation. De plus, il constitue un élément qui aide à la construction de la vulnérabilité sociale et territoriale des petits producteurs et des communautés rurales. La grande productivité des sols pampéens et la conjoncture favorable du soja sur le marché international, du fait de la demande d'aliments et de biocarburants, produisent un important revenu différentiel. Grâce à la rentabilité actuelle de la production agricole, le campo est devenu un secteur attrayant pour l'investissement des capitaux provenant d'autres activités, c'est-à-dire un espace propice à la spéculation financière.

\section{Concentration de la terre et concentration du capital dans la production}

11 D'après une estimation du revenu des terres, réalisée par Azcuy Ameghino (2007), le revenu net de ces terres est d'environ 4,5 milliards de dollars et si on y ajoute le revenu en élevage, il atteindrait 8 milliards. Si l'on considère qu'il y a " une relation directe entre la distribution de la propriété de la terre et celle du revenu » et que seuls $10 \%$ des propriétaires contrôlent la moitié de la superficie agricole, on pourrait donc déterminer que « ce pourcentage de propriétaires fonciers perçoit quelque 4 milliards de dollars à titre de rétribution générée par l'utilisation des terres productives dont ils sont propriétaires » (Azcuy Ameghino, 2007, p. 131).

12 La production à grande échelle - concentration de l'exploitation économique des terres agricoles - permet d'augmenter la rentabilité, en diminuant les coûts de production. Cette diminution est également due à la croissante capacité de négociation, avec la monopolisation de la production agricole. Il en résulte donc un facteur menaçant pour la petite et moyenne exploitation agricole. Cependant, même si on affirme qu'il y a eu, pendant ces dernières années, un processus de concentration des terres, tant de la propriété de la terre que de son utilisation, le débat reste fondé sur l'information disponible vers la fin des années 1980 et le début des années 1990. Néanmoins, les données issues des recensements des années 1988 et 2002 confirment que le nombre d'exploitations agricoles du pays a diminué de $25 \%$ sur cette période. En même temps, la moyenne de la superficie des exploitations a augmenté progressivement. De plus, la 
valeur marchande des terres a subi une extraordinaire augmentation depuis la dévaluation ${ }^{15}$. À tel point que le prix de l'hectare peut valoir - aujourd'hui- dans la Pampa humide près de 12000 dollars, c'est-à-dire qu'il dépasse les valeurs des meilleures terres productives des États-Unis (Cufré, 2008, p. 2).

\section{Les acteurs de toujours et les nouveaux acteurs}

13 Depuis le milieu des années 1990, dans un contexte où les prix internationaux des commodities augmentaient rapidement et où une politique nationale maintenait le dollar à un prix élevé, le petit et moyen producteur du secteur central ${ }^{16}$ de la région pampéenne a dû choisir entre deux alternatives : travailler la terre ou vivre de la rente foncière. La montée exceptionnelle des prix de la terre a poussé la plupart à vendre. Certains ont investi cet argent dans les commerces de leur village. D'autres ont acheté des biens immobiliers dans ces mêmes villages ou dans de grandes capitales, afin de les louer - ce qui explique en bonne mesure, le boom de la construction dans ces villes et capitales provinciales. D'autres encore ont préféré affermer. C'est ainsi que de nouveaux agents agricoles, dont on méconnaissait la puissance, sont apparus. Ces agents économiques sont les bailleurs, les acheteurs investisseurs, les anciens producteurs désormais devenus des rentiers-commerçants ${ }^{17}$. Et, ce sont eux qui ont été les acteurs principaux du lockout ${ }^{18}$ aux proportions exceptionnelles, de mars 2008 (Zaiat, 2008). L'augmentation du revenu et de la valeur des terres a confronté les petits propriétaires de 50 à 100 hectares à un dilemme : soit percevoir une importante rente annuelle en affermant pour du blé ou du soja, soit produire afin d'obtenir plus de profits mais en prenant tous les risques que l'activité agricole entraîne. À cette échelle, les décisions ont été réparties. La plupart des propriétaires de 500 à 1000 hectares ont préféré une rente fixe et extraordinaire. L'investisseur externalise le travail d'ensemencement ainsi que celui de la récolte. En somme, ce n'est pas lui qui fait la partie la plus difficile du travail, mais l'entrepreneur, propriétaire des machines agricoles, qui s'en occupe. Il y a donc un troisième acteur qui va tirer ses bénéfices de la même unité de terre. C'est ainsi que de grands, petits et moyens propriétaires, devenus rentiers, ont eu la possibilité d'investir l'argent perçu par les baux dans un fonds d'investissement : les pools de semailles ${ }^{19}$ afin d'accroître encore plus la rentabilité, sans participer directement à aucune tâche agricole. À tous ces acteurs, on doit ajouter les propriétaires-producteurs qui prennent des terres ${ }^{20}$. Actuellement, $49,5 \%$ de la superficie de la région pampéenne (36,8 millions d'hectares) a été prise à bail (Recensement national agricole 2002) ${ }^{21}$.

14 Les grandes entreprises exportatrices de céréales et d'huiles, dont les plus importantes sont Cargill SACI, Bunge Argentina SA, Nidera SA, LCD Argentina (Dreyfus) et Huilerie General Deheza (Famille Urquía, Córdoba) ${ }^{22}$, jouent également un rôle décisif dans la structuration du modèle de sojisation. Leurs terminus portuaires à Rosario, San Martín et Arroyo Seco (province de Santa Fe), à Buenos Aires ou Quequén (province de Buenos Aires) et les lignes de transport de charge par voies ferrées, sont structurantes du contenu du territoire ${ }^{23}$. Tout cet agro-business a été motivé par Monsanto (États-Unis), l'entreprise de grains la plus importante au monde. Cette entreprise vend une grande quantité d'agrochimiques (la plupart des agrotoxiques) nécessaires à l'extermination des mauvaises herbes, que le semis direct n'élimine pas. De plus, Monsanto crée les OGM résistant aux pesticides ${ }^{24}$ et herbicides, qui assurent la dépendance des acheteurs, et par conséquent garantissent sa propre production (chaque grain OGM appartient à 
son inventeur, ce grain est breveté et protégé par la réglementation de l'Organisation Mondiale du Commerce). Monsanto a créé un paquet technologique - soja RR (Roundup Reddy) - composé de grains génétiquement modifiés qui résistent à l'herbicide glyphosate et qui sont associés au semis direct ${ }^{25}$. D'ailleurs, Monsanto a acheté toutes les entreprises productrices de grains, ce qui a obligé les agriculteurs à lui payer des redevances. En Inde, 100 \% des grains sont transgéniques. En Argentine, 90 \% de la production de soja est transgénique. Depuis 2005, Monsanto fait pression pour obtenir les redevances en Argentine, malgré la promesse qu'elle avait faite, il y a quelques années, de "ne pas les faire payer ${ }^{26}$ " (Robin, 2008 ; Cóccaro et al., 2006). Actuellement, dix entreprises dans le monde entier, contrôlent $32 \%$ du marché des grains commerciaux (dont la valeur estimée est de 23 milliards de dollars) et la totalité du marché des grains OGM. Elles contrôlent également le marché de l'agrochimie et des pesticides au niveau mondial ${ }^{27}$.

Dans ce contexte, le rôle de l'État est limité à la préservation de la liberté de marché, en évitant l'établissement de toute politique économique capable de perturber son fonctionnement naturel. Ainsi, dans un effort pour situer de manière interprétative le processus de sojisation, Azcuy Ameghino (2004) et Gómez (2003) ${ }^{28}$ énumèrent les politiques établies depuis les présupposés néolibéraux qui ont eu un impact sur le secteur agricole pampéen et fournissent les orientations explicatives de la crise actuelle

16 - La réduction radicale de l'État et le changement de son rôle s'opèrent à travers un processus de décentralisation des activités de l'État National vers les provinces et des provinces aux communes. Ces politiques obligent les provinces et les communes à résoudre des problèmes attenants à la santé et à l'éducation, entre autres. Par ailleurs, la Junta Reguladora de Granos (JRG) y la Junta Nacional de Carnes (JNC) ${ }^{29}$ et des institutions telles que l'INTA ${ }^{30}$ vivent actuellement une situation critique à cause de leur définancement.

17 - La privatisation des entreprises de l'État laisse dans des mains privées la production de carburant, le contrôle des routes nationales, des ports et des chemins de fer. Par conséquent, les coûts de transport, de récolte et d'ensemencement augmentent.

18 - La déréglementation des marchés intérieur et extérieur est en rapport avec la suppression de la JRG et la JNC. C'est la suppression des obstacles au commerce, l'ouverture au marché mondial et l'impulsion à l'arrivée d'entreprises transnationales. Dans le cas de l'agriculture pampéenne, ces entreprises deviennent des monopoles de distribution de semences, de produits agrochimiques et de technologie en général.

Voici, en termes généraux, le profil des acteurs hégémoniques de la construction du modèle de sojisation qui reflète une façon de penser le territoire et qui fonde les bases pour l'analyse portant sur la construction de la vulnérabilité socio-territoriale des petits producteurs et des communautés rurales.

\section{Le territoire : agriculturisation et sojisation}

Quant au processus d'agriculturisation, il ne suscite pas trop de désaccords. Les controverses tournent autour de l'impact social et environnemental d'un système de monoculture généralisé. La sojisation, dans un contexte d'agriculturisation, a eu des répercussions lors de l'insertion de l'Argentine dans le marché mondial. L'utilisation de biotechnologies engendrées dans les pays centraux, influence l'insertion dépendante 
du pays dans la division internationale du travail. Depuis les années 1970, un important processus d'agriculturisation des terres de la pampa argentine est effectué. Le rôle prépondérant des cultures de soja est en augmentation progressive " jusqu'à la moitié des années 1990, période à partir de laquelle il bondit pour atteindre les hauts niveaux actuels » (Azcuy-Ameghino, 2004, p. 66). Il passe en effet de 5007000 ha de superficie semée pendant la campagne 1991-1992, à plus de 16000000 ha pendant la campagne 2006-2007, avec une récolte de 43 millions de tonnes de soja (journal Clarín, 2007). En 15 ans, la superficie de cultures de soja augmente de $319 \%$ et sa production, de $241 \%$.

Dans le but de montrer une manière de penser la production du territoire, à partir des présupposés néolibéraux, l'élargissement de la frontière agricole et la pampéanisation à travers la sojisation sont plus intéressants que l'augmentation de la production due au rendement dans la région pampéenne proprement dite. En tout cas, il s'agit d'abord, de l'élargissement de l'homogénéisation, puis de l'intensification de l'homogénéisation.

Les figures 2 et 3 montrent deux aspects de ce processus : le pourcentage des terres agricoles consacrées à la culture du soja selon les recensements nationaux agricoles de 1998 et de 2002, et le rapport entre le pourcentage des terres destinées à l'agriculture et celui des terres semées avec du soja, détaillé selon les départements provinciaux ${ }^{31}$, d'après le Recensement national agricole 2002. Dans la même période, l'augmentation de la superficie semée avec du blé n'a été que de $+1 \%$, avec une production accrue de 22 $\%$. La superficie semée en maïs a augmenté de $12 \%$, avec une augmentation de production de $72 \%$. La production de soja pendant les campagnes de 1985-1989 a été de 8173340 tonnes avec un rendement de $2 \mathrm{t} / \mathrm{ha}$ en moyenne, tandis que la campagne de 2006-2007 montre un rendement de 2,65 t/ha.

Figure 2 : Part des terres agricoles destinées à la culture du soja en Argentine en 1988 et en 2002 Part of cultivated land used for soy crops in Argentina in 1988 and in 2002

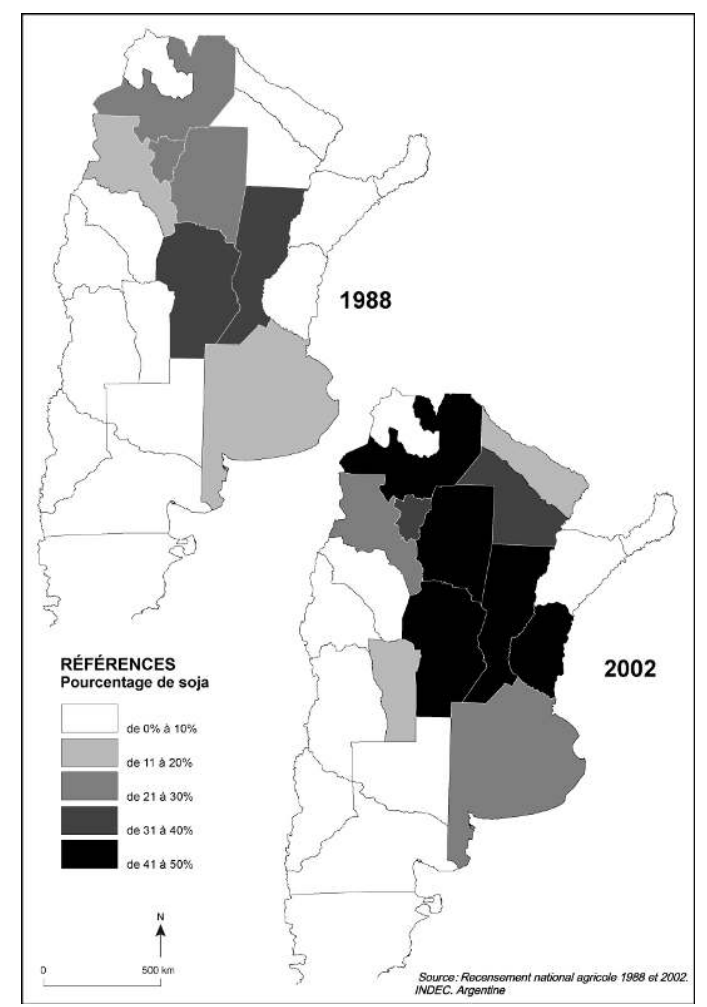


Ce processus, en termes de paquets technologiques, exprime une tendance à l'homogénéisation, tout en camouflant les différences sociales, territoriales et environnementales qu'il entraîne. L'augmentation, en tonnes, du soja produit est due à deux éléments essentiels : a) l'intensification et la productivité et, b) l'expansion territoriale de sa production. En ce qui concerne le premier élément, l'introduction du soja transgénique dans le pays a impliqué un changement des caractéristiques de la production qui a modifié et qui modifie encore la provision des semences, le semis direct et les produits agrochimiques. C'est la conjonction de ces éléments ainsi que l'adaptabilité des semences transgéniques dans différentes régions qui a permis la forte croissance de la productivité du soja. Quant au deuxième élément énoncé - celui de l'expansion territoriale du soja - la superficie semée de cet oléagineux s'est élargie par : a) le remplacement d'autres céréales et d'autres oléagineux qui étaient cultivés dans la région pampéenne; b) le remplacement de l'élevage par la culture de $\operatorname{soja}^{32}$; c) le remplacement des productions régionales ${ }^{33}$ et $\mathrm{d}$ ) le défrichement des champs aussi bien dans des provinces extra-pampéennes ${ }^{34}$ que dans des régions pampéennes marginales. De cette manière, la culture du soja traverse de nombreux biomes du pays et homogénéise le type et le système de production. La figure 4 montre le rapport entre les biomes, profondément modifiés aujourd'hui, et les terres agricoles consacrées à la culture du soja selon le Recensement national de l'agriculture de 2002.

Figure 3 : Terres agricoles et terres cultivées en soja en Argentine en 2002 Cultivated lands and soy crops in Argentina in 2002

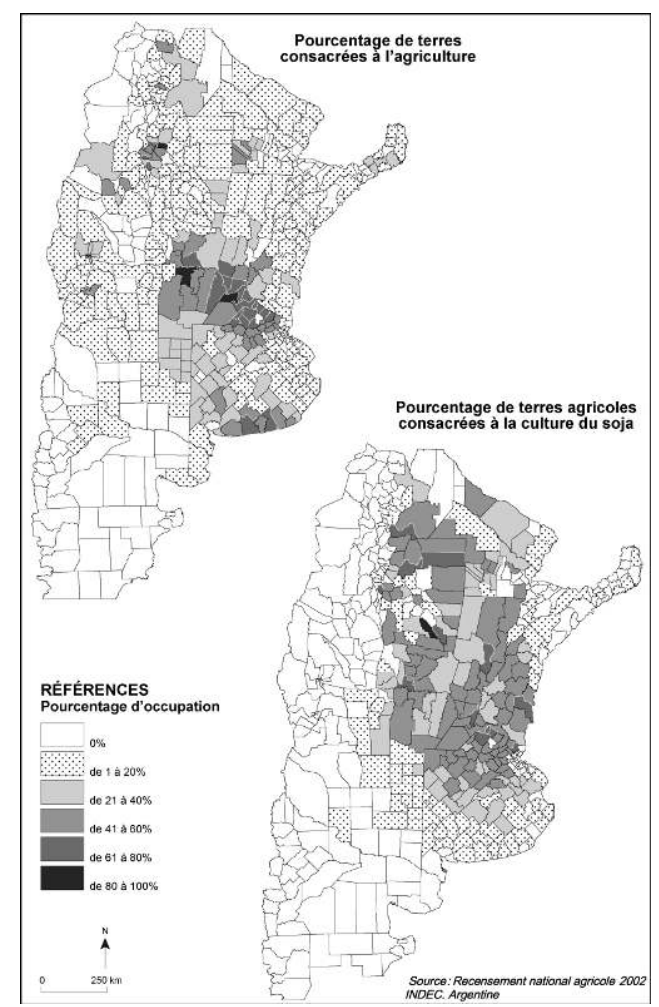


Figure 4 : Relation entre les biomes de l'Argentine et la culture du soja en 2002 Eco regions and soy crops in Argentina in 2002

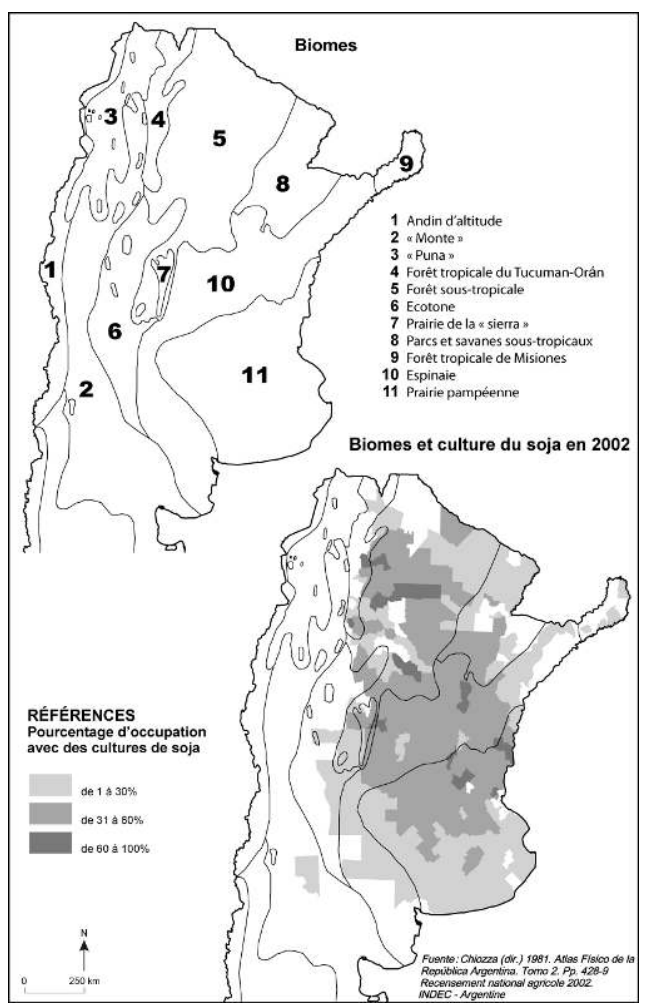

\section{Le territoire : vulnérabilité sociale et environnementale de la sojisation}

Les impacts socio-environnementaux du développement du capitalisme agricole dans la région pampéenne ont soulevé de nombreuses controverses. L'impact social dépend du présupposé de la déconcentration de la propriété. En effet, si la propriété du territoire permet de percevoir le revenu à qui la détient, le processus de déconcentration accorderait à un plus grand nombre de personnes, l'accès aux bénéfices du secteur agricole. La situation est à l'inverse dans le cadre d'un processus de concentration de la terre et de la production, car les bénéfices ne reviennent qu'à un nombre réduit de personnes.

\section{Des arguments « légitimant » le modèle}

Ceux qui préconisent le modèle de sojisation le considèrent plus démocratique, car il entraîne le développement d'un "marché dynamique des terres et des services permettant de faire de l'agriculture sans terre par la disposition d'un schéma extraordinairement démocratique d'accès aux ressources " (Grobocopatel, 2005). En ce qui concerne le chômage et l'expulsion de salariés ruraux, ils préconisent le changement des schémas traditionnels de calcul du taux de chômage car ils soutiennent qu'il y a un changement du tissu productif. Les activités dans des secteurs sans rapport direct avec la production du sol se multiplient, mais elles restent, néanmoins dépendantes de celle-ci. Par conséquent, la synergie du système de 
production actuel augmente les pourcentages d'emploi et " inclut des millions de personnes, directement ou indirectement liées à la chaîne agro-alimentaire, qui sont partie intégrante de la communauté rurale argentine, éparpillée dans des milliers de villages ou de villes de notre Argentine [...]. Dans ces villes et villages, il y a énormément d'emplois associés à la couverture des besoins des "chacrers ${ }^{35}$ " et de leurs familles... ", même si " il y a des transformations douloureuses en cours " (Seminario, 2001). Dans la même ligne de pensée, Trucco (2004) affirme que le système « favorise l'inclusion, mais il faut aider les gens à s'en rendre compte, à reconnaître les opportunités ». « La concurrence est la lutte de tous les jours, celle qui nous réveille et qui nous demande d'innover, afin de maintenir la rentabilité. Grâce à la concurrence, les gens gagnent, car ils obtiennent plus d'aliments à moindre coût, même si parfois, certains d'entre nous restent à côté du chemin » (Trucco, 2000 cité par Pengue, 2005, p. 98).

Par ailleurs, ils nient la non durabilité environnementale des monocultures, par le fait que, le système de labour zéro diminue l'érosion du sol, en laissant le chaume qui le protège de la pluie et du vent. Le fait de laisser les chaumes sur le sol, permet de mieux maintenir ses propriétés productives et, conjointement avec une rotation adéquate des cultures et la fertilisation des sols, on obtiendra non seulement plus de rendements économiques, mais on assurera aussi la parfaite conservation de la ressource sol, voire son enrichissement, en vue de son utilisation future (Lorenzatti entretien au journal Clarín, 24 août 2006). L'éditorial du journal La Nación du 4 novembre 2006 affirme que « de toutes les ressources qui peuvent passer à dans des mains étrangères, celle qui doit le moins nous inquiéter est la terre, de par son caractère d'immobilité ». Et, en ce qui concerne l'impact sur la santé des gens, ils soutiennent qu'il n'y a pas de recherches " scientifiques » qui l'attestent et, par conséquent, elles ne doivent pas être prises en considération. Ainsi Huergo (2006) écrit « [...] nous avons découvert aussi, sans aucun aval de recherche sérieuse, que le soja provoque chez les filles une première menstruation précoce. Qu'il peut faire pousser de petits seins chez les garçons, que les femmes enceintes et les bébés ne doivent pas en consommer. J'ai même entendu cette semaine, sur une radio leader, un médecin qui soutenait ces stupidités avec une totale fluidité et ignorance. ».

Il est courant d'écouter, chez les préconisateurs de ce modèle, que l'on doit accepter les changements et s'y adapter afin de ne pas renoncer au progrès. "Si nous ne nous "rendons pas compte" que les changements profitent à certains et portent préjudice à d'autres, et si l'État et les institutions ne se consacrent qu'à défendre les perdants, on finit par préserver le statu quo et par renoncer au progrès. » (Trucco, 2003).

\section{Des arguments « résistant » au modèle}

28 Cependant, il est possible d'énumérer, à présent, de nombreux impacts négatifs (ou " des effets collatéraux non désirés " du système, dans la dénomination de ceux qui légitiment le modèle de sojisation). Plusieurs d'entre eux, que les auteurs de cet article partagent, s'opposent aux arguments favorables de la section précédente :

- La diminution de la population économiquement active (PEA) rurale : la technification progressive des activités agricoles implique l'expulsion des travailleurs salariés ruraux. Dans le Sud de la province de Córdoba, des études ont quantifié cette réduction (Agüero et al., 2004). En effet, à l'aire d'influence des dix communes du Sud de Córdoba, la PEA 
rurale a diminué de 36,2 \% pendant la période 1994-2000. D'autre part, la région pampéenne a perdu plus de $30 \%$ des exploitations agricoles avec un processus corrélatif de concentration des revenus dans ce secteur.

- Le surgissement d'un modèle d'agriculture sans agriculteurs par le faible volume de main-d'œuvre nécessaire aux nouveaux systèmes de culture et la haute rentabilité de la production à échelle. Ceci a facilité l'expulsion des petits producteurs et la dépendance de moyens producteurs à un paquet technologique, ce qui limite leur possibilité de prendre des décisions concernant leur production et la manière dont ils doivent la réaliser (Cóccaro et al., 2006).

31 - L'existence d'innombrables villages ruraux qui se trouvent en voie de disparition ou qui ont déjà disparu. La géographe Marcela Benítez a réalisé une étude concernant les villages en risque $^{36}$, en comparant les données des recensements de 1980, 1991 et 2001. Elle a ainsi observé qu'il existe dans le pays, 602 villages de moins de 2000 habitants en risque de disparition. La plupart d'entre eux se situent dans la région pampéenne qui, en 2001, comptait 488 villages sans croissance ou en voie de disparition. À ce sujet, Carola Pessino, secrétaire à l'Équité fiscale pendant le gouvernement de Menem (1989-1999) a affirmé qu'" avant, grâce à des subventions de l'État, on entretenait des sources [de travail]inefficaces, donc les gens demeuraient toute leur vie dans un endroit déterminé. Maintenant, c'est dur, mais ils devront déménager là où il y a du travail » (journal Clarín $)^{37}$. Ce processus, tout comme les deux points précédents, explique la continuité de l'exode rural.

32 - L'extraction minière des pampas, provoquée non seulement par l'exportation des grains, mais aussi par l'exportation parallèle des nutriments du sol argentin. «Tous les ans, l'Argentine exporte des millions de tonnes de nutriments naturels qui ne sont pas récupérés de façon durable. Rien qu'en tenant compte de ses principales cultures, le pays exporte annuellement avec ses grains - et gratuitement ! - environ 3,5 millions de tonnes de nutriments. Le soja, moteur de l'agriculture exportatrice argentine, représente presque $50 \%$ de ce chiffre " (Pengue, 2003). En ce qui concerne la dénonciation du développement de ce type d'agriculture minière, paradoxalement, la photo 1 présente la publicité de Fertilizar (Association civile) qui vise à se différencier nettement de l'activité minière, si contestée de nos jours. 
Photo 1 : Publicité de Fertilizar Asociación Civil, parue dans le journal La Nación Advertising for Fertilizar Asociación Civil, issued in the La Nación paper

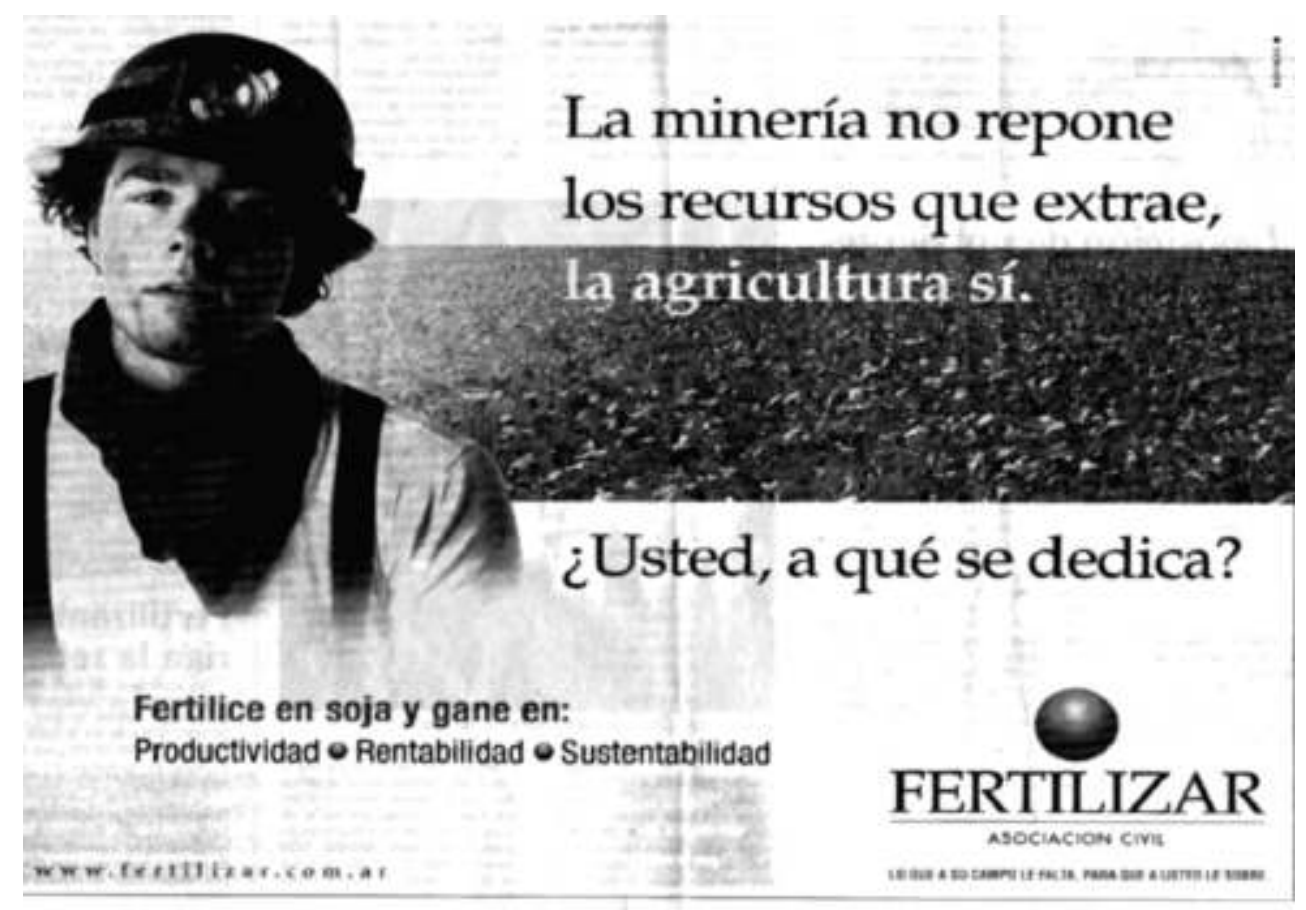

33 - Un accroissement de la quantité de produits agrochimiques employés dans la production agricole, avec le risque potentiel de nuire à la santé et de polluer les nappes phréatiques ou les cours d'eau ${ }^{38}$.

34 - L'impact produit par l'introduction de transgéniques. Il est vrai que les études concernant l'impact de la consommation de produits transgéniques sur les êtres humains, ainsi que l'impact provoqué dans divers écosystèmes par l'introduction de ces organismes génétiquement modifiés sont encore naissantes. Pourtant, elles alertent tout de même sur les modifications que ces produits entraînent, notamment chez les moins de quatre ans ${ }^{39}$.

- La perte de la souveraineté alimentaire. Comme il a déjà été signalé, le processus de sojisation a eu lieu au détriment d'autres activités pampéennes et extrapampéennes. " La forte concentration vers le soja a produit un important déplacement d'autres productions, tout en érodant une base productive et diverse. Toutes les économies régionales, ainsi que beaucoup d'autres productions, ont vu leurs espaces occupés par l'avancée du soja »(Pengue, 2005, p. 84). Sur la figure 5 est présenté un panorama général des mouvements et des résistances sociaux face à l'expansion du processus de sojisation en Argentine ${ }^{40}$. 
Figure 5 : Mouvements et résistances sociales contre l'expansion du soja en Argentine Social resistance and movements against the spread of soy crops in Argentina

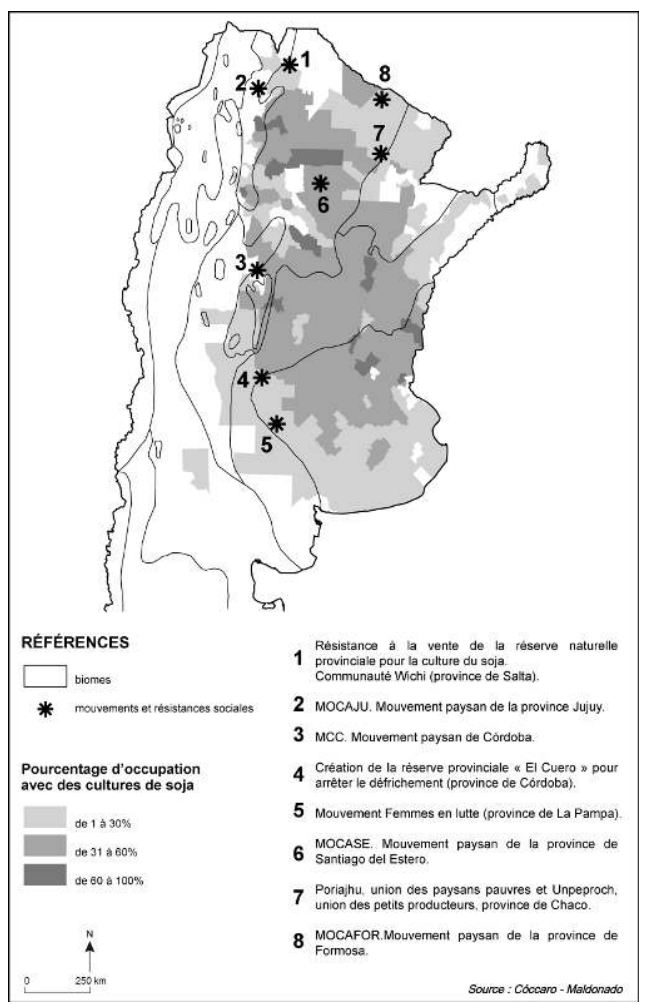

36 - La perte de biodiversité et de socio-diversité, résultat du défrichement, de l'expulsion de communautés aborigènes de leurs terres, et de la modification de la structure sociale agricole $^{41}$.

- L'accroissement de la dépendance du producteur et du pays, dans deux sens. D'un côté, les producteurs sont à la merci des variations des prix internationaux du seul grain qu'ils produisent. D’un autre côté, ils dépendent de l'approvisionnement du paquet technologique, qui appartient à des multinationales.

38 - Des problèmes environnementaux associés à : a) l'intensification des inondations dans certaines régions ${ }^{42}$; b) l'intensification $d u$ processus d'érosion des rives des fleuves et ruisseaux (Cholaky et al, 2005) provoqué, entre autres, par l'augmentation de l'écoulement superficiel de l'eau, le compactage du sol (Cisneros et al., 2004 ; Cisneros et $a \mathrm{l}, 2005)$ et sa mauvaise gestion ; et c) la perte du sol par l'érosion éolienne, ainsi que par le processus de défrichement incontrôlé.

39 C'est-à-dire que « ce qui est arrivé pendant les dernières années dans le secteur agricole argentin résulte, en grande partie, de l'articulation des tendances de longue durée du développement du capitalisme - dépendant et de faible intensité - avec une politique économique qui a stimulé, accéléré et aiguisé les lois de l'accumulationdésaccumulation du capital, dont les effets socio-économiques ont atteint des niveaux d'extrême difficulté » (Azcuy Ameghino, 2004, p. 71). Le problème n'est pas le soja, mais les lois économiques et les politiques publiques. Le soja représente le territoire en tant que marchandise, selon les principes néolibéraux. 


\section{D'autres chemins possibles : le territoire comme patrimoine social commun}

\section{Le territoire marchandise}

Dans le secteur agricole, historiquement, les variables d'ajustement en cas de crise ont toujours été les salariés ruraux ${ }^{43}$ ou les petits et moyens « chacareros ». Cependant, « la solution au problème du travail [...] n'est pas de sacrifier les facteurs humains de la production mais d'accomplir le mot d'ordre : "La terre doit être un bien de travail et non pas de revenu" "(Frigerio, 1953, p. 113). Dans cette même ligne de pensée, quelques processus différents de réforme agraire de portée sociale et territoriale ont été développés en Amérique Latine ${ }^{44}$. Par définition, une réforme agraire est complexe, puisqu'elle « ne peut pas se restreindre à la terre (elle ne serait que foncière) ou à la production (elle ne serait qu'agricole) ; elle est dite agraire justement parce qu'elle doit inclure tout cela et s'étendre sur tout le contexte social " (Giberti, 1998, p. 67). L'Argentine n'a pas développé de processus de réforme agraire. En effet, même si quelques lois ou programmes destinés à protéger les petits propriétaires et fermiers ont existé, ils ont toujours été loin de constituer un véritable processus de réforme agraire.

41 Afin de renverser la situation traversée par le secteur rural aujourd'hui, caractérisée par un processus d'exclusion sociale très net, Azcuy Ameghino (2004) présente deux perspectives futures possibles : premièrement, rénover l'intervention de l'État en économie agraire, avec la recréation de la Junta Reguladora de Granos (Comité national pour les grains) et la Junta Nacional de Carnes (Comité national pour la viande), sous un modèle de planification de la production agricole visant à un développement national et régional, intégré et harmonieux; deuxièmement, restreindre le pouvoir des oligopoles d'exportation, de l'agro-industrie et du commerce intérieur, limiter le revenu foncier et les surfaces à louer à de gros capitaux. La lutte actuelle pour une réforme agraire implique, non seulement de faire face à des conflits d'intérêts avec les gros propriétaires fonciers nationaux historiquement affermis, mais encore avec les groupes investisseurs qui ont trouvé dans le secteur agricole un milieu rentable pour leurs investissements et spéculations. Elle implique également des conflits avec les entreprises transnationales qui ont déployé un système agro-industriel extrêmement dépendant d'elles-mêmes.

La question que l'on se pose ici est donc : quel type de connaissance géographique présuppose $t$-on afin d'argumenter une solution alternative pour réguler l'espacemarchandise, inspirée par une éthique de responsabilité sociale et d'apport au bien public? Si le désir est légitime (nous en sommes persuadés) le premier pas est d'excercer la capacité d'interpellation et la capacité critique nécessaires pour évaluer la production de connaissances géographiques et théoriser afin de représenter, et représenter afin d'intervenir dans la formulation de principes envisagés sous cette perspective. Si on ne peut pas changer le monde maintenant, il est possible de commencer par changer la façon de l'observer. 


\section{Le territoire : patrimoine social commun}

43 universellement acceptée. Elle est justifiée par le fait qu'il s'agit d'un bénéfice provenant $\mathrm{du}$ milieu naturel et non pas $\mathrm{du}$ prix à des efforts individuels d'investissement, de génie ou de travail, qui doivent être à leur tour rétribués de manière équitable. Quel pourcentage du soja produit en une unité d'exploitation est le résultat d'effort d'investissement en tout sens et quel pourcentage est le produit de la capacité productive naturelle du sol ? La propriété de la terre ou la faculté de la louer n'implique pas l'appropriation du sol par le propriétaire-producteur ni par le locataire. La propriété de la terre n'est pas associée à la propriété du sol. Le sol, en tant que ressource naturelle est patrimoine social commun. Les rétentions ou droits d'exportation historiques permettent que la société s'approprie une partie de la richesse du pays. La société peut ainsi obtenir les biens de production primaire (y compris les dérivés du pétrole et ses utilisations internes) à des prix inférieurs à ceux qui résulteraient du taux de change en vigueur. Le fait qu'aujourd'hui celui-ci soit élevé est nécessaire pour le développement du marché intérieur. C'est une caractéristique spéciale de l'Argentine, due à l'étroite relation existant entre les prix des produits agricoles et énergétiques d'exportation et un panier type de consommation populaire.

C'est la juste distinction entre producteurs de différents secteurs et des conditions pour une application des impôts plus équitable et socialement responsable. On ne doit pas oublier l'articulation des intérêts individuels légitimes avec ceux de la société dans son ensemble, notamment ceux des plus défavorisés. Dans ce contexte, il faut réussir un large accord concernant l'utilisation d'instruments de change, de fiscalité et de crédit 
(Plan Fénix, 2003-2008). Le problème central implique la distribution des revenus extraordinaires provenant de la culture du sol et la transformation d'un modèle productif de pays pour peu de mains en un modèle productif pour tous.

Sous une perspective critique de responsabilité sociale et d'apport au bien public, la monoculture du soja et le processus de sojisation nous interpellent et nous mènent à la recherche d'une façon alternative de penser le territoire. À partir de cette " situationnalité "/« contextualité, il faut construire des catégories d'analyse nous permettant de concevoir et de produire un espace plus humanisé. « Un espace, en tant qu'instrument de reproduction de vie, et non pas la marchandise travaillée par une autre marchandise, l'homme fétichisé » (Santos, 2002, p. 35). Pourtant, les catégories d'analyse, toujours tributaires de leur relativité historique, ne sont pas seulement légitimées par la solidité et la rigueur académique de leur conception. Le territoire en tant que produit et producteur de la société apparaît ainsi comme une catégorie irréprochable dans beaucoup de discours géographiques. Cependant, lorsqu'elle est traversée par un aspect multidimensionnel idéologique et éthique, elle devient un instrument d'analyse et d'interpétation de différentes représentations construites de la réalité conditionnant les politiques d'intervention (Maldonado et Cóccaro, 2008). Sur le tableau 1 sont présentées des façons alternatives de penser le territoire à partir de différents présupposés.

Tableau 1 : Le territoire comme produit social Territory as a social production

\section{TERRITOIRE COMME PRODUIT SOCIAL}

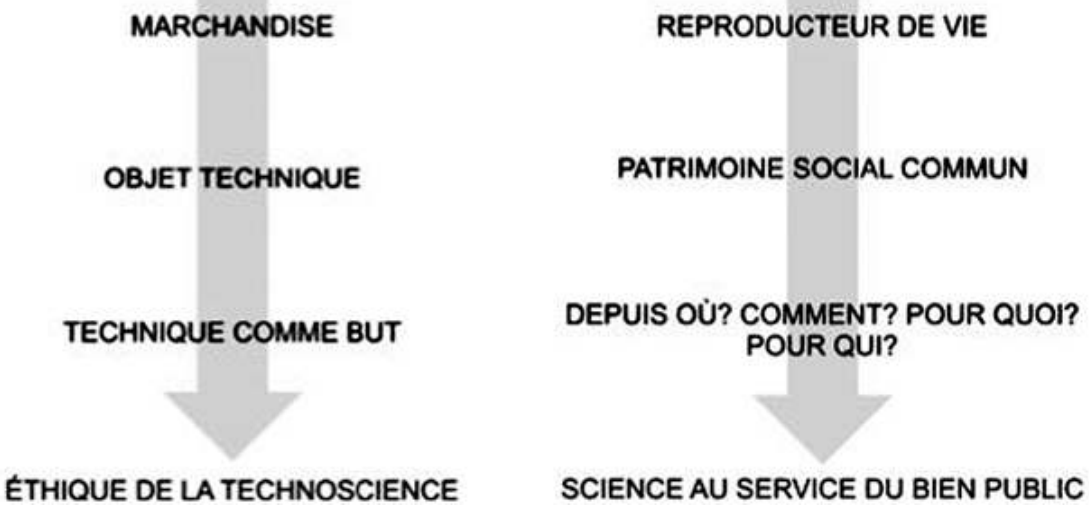

\section{Des propositions d'espaces}

Il s'agit ici de proposer des espaces pour une analyse politique et éthique d'après la perspective de responsabilité sociale et d'apport au bien public et des approches théoriques et méthodologiques pour comprendre la vulnérabilité sociale et environnementale du secteur agricole pampéen dans le contexte du développement du capitalisme agricole. Le chemin parcouru jusqu'à présent dans ce travail constitue un trajet incontournable pour comprendre la vulnérabilité socio-territoriale du secteur agricole pampéen, dans le contexte du développement du capitalisme agricole. On trouve aussi, un peu plus loin, les jalons pour la construction des indicateurs de 
vulnérabilité. Cette vunérabilité socio-environnementale repose sur les inégalités socio-territoriales, c'est pourquoi, de même que celles-ci, elle ne peut pas être comprise à moins d'être replacée dans le contexte historique et social d'où elle a émergé. Aussi, considère-t-on que la vulnérabilité se construit historiquement et socialement. Elle est en rapport avec la géographie historique de l'accumulation du capital.

Définir les facteurs qui rendent les personnes (dans ce cas, les producteurs agricoles) plus ou moins vulnérables, implique la mise en jeu d'un devoir être de la situation du campo et de sa fonction sociale. Ainsi, selon les principes proposés dans le paragraphe précédent, le sol argentin accomplit l'inéluctable rôle social de contribuer au développement du pays. Par conséquent, il ne peut pas être seulement évalué par son niveau de productivité, mais également et fondamentalement, par sa contribution à un projet de pays ayant comme priorité le bien-être social. Le fait de continuer le processus de sojisation, sans réglementations sérieuses, implique ne penser qu'au court terme. Il faut mettre fin au modèle d'agriculture sans agriculteurs, dirigé par un système de monoculture, et parallèlement, promouvoir des programmes de développement d'agriculture familiale ${ }^{45}$, dans de petites et moyennes exploitations à production diversifiée et durable ${ }^{46}$. Selon ces principes, tout processus de concentration de la propriété, concentration économique, expulsion de petits et moyens producteurs, monocultures de tout type, entre autres, accentuent la vulnérabilité socio-territoriale, non seulement des producteurs familiaux, mais aussi des régions intimement liées au secteur agricole. Ainsi, la construction d'indicateurs constitue une issue pour déterminer les niveaux de vulnérabilité et un outil pour l'intervention sociale.

\section{Modèle pour la construction des critères pour l'élaboration des indicateurs}

Nous avons exposé notre travail pour la construction d'une structure conceptuelle cohérente, selon une géographie critique, afin d'avancer dans la compréhension du processus continuel de construction de l'espace. Celle-ci provient d'une série d'apports réalisés par différents auteurs pour l'analyse de problématiques situées. En ce qui concerne la construction d'un modèle de médiation entre la " réalité » et les réflexions méthodologiques, qui soit pertinent, cohérent et opérationnel, nous coïncidons avec María Laura Silveira qui introduit le concept de situation géographique en tant qu'outil servant à analyser le territoire (Silveira, 2001). Elle définit la situation en tant que localisation matérielle et relationnelle qui peut être conçue comme une partie de l'histoire présente et qui permettrait de reconnaître l'impact d'un ensemble d'événements sur un lieu, contenant des actions et des objets qui aident à sa construction et reconstruction de manière à ce qu'elle redéfinisse constamment la spécificité du lieu $(1999,2001)$. L'accent est mis sur les systèmes normatifs qui régulent les actions, car la densité normative différentielle des lieux est un antécédent et elle crée donc la segmentation du territoire (2003). Dans le concept territoire utilisé on trouve la bonne catégorie pour : analyser les existences sur le territoire à un moment donné, observer la dynamique de l'utilisation du territoire à travers la rationalité hégémonique actuelle et proposer de quelle manière on devrait s'en servir (2007). Ainsi, le territoire en tant que contenu est l'expression de verticalités qui déterminent des points et des zones d'action des agents hégémoniques. Pourtant, il exprime également les horizontalités définies comme les secteurs où se produit la plupart du travail social 
des agents non hégémoniques qui travaillent dans la contiguïté. Parfois, elles utilisent les divisions territoriales du travail ayant eu lieu dans des périodes précédentes, où il existait autant d'actions de subordination que de résistance. Ces verticalités et horizontalités n'expriment pas de dualisme mais une situation impure. Des objets qui se présentent, dans un premier temps, comme des horizontalités peuvent, plus tard, devenir des verticalités. Ce sont des catégories d'analyse qui aident à la compréhension de la situation impure et à dévoiler les logiques subjacentes. Elles font face au défi de différencier des échelles d'origine et des échelles d'impact.

51 Le processus de sojisation rend visibles les verticalités et les horizontalités et met en évidence le pouvoir qu'ont les entreprises pour produire un territoire corporatif et une utilisation corporative du territoire (Silveira, 2008). Il existe une image hégémonique $\mathrm{du}$ territoire dont l'analyse décrit son fonctionnement. Mais, il faut être attentif aux tendances d'utilisation du territoire : comment a-t-il été utilisé ? Comment est-il utilisé ? Comment pourrait-il être utilisé ? Comment devrait-il être utilisé selon une perspective de responsabilité sociale et d'apport au bien public ?C'est ainsi que la vulnérabilité sociale et territoriale du secteur agricole pampéen, face à l'expansion de la sojisation est déjà comprise dans le territoire qui se porte modèle producteur des inégalités et élément fondateur des vulnérabilités constituantes et conditionnelles. Dans ce cas, la vulnérabilité sociale doit être considérée comme le résultat de la relation entre la disponibilité et la capacité de mobilisation des actifs sociaux ${ }^{47}$, et la structure d'opportunités ${ }^{48}$ dans une formation sociale et spatiale "située " (Filgueira, 2006). La construction d'un critère pour l'élaboration d'indicateurs de vulnérabilité socioterritoriale pour le monde rural, spécifiquement dans la région agricole pampéenne, devrait considérer les échelles d'analyse d'approximation et les dimensions suivantes :

52 - À macro-échelle : l'influence du contexte néolibéral à travers l'expression et la matérialisation de ses principes.

53 - À méso-échelle : comprendre et conceptualiser la formation, l'utilisation et la reproduction des actifs sociaux (Les caractéristiques du modèle rural ; l'analyse du lien entre la production et les vocations agro-écologiques de la région).

54 - À micro-échelle : l'analyse des processus endogènes (caractéristiques internes des productions agricoles) liés à leur capacité de reproduction et à leur type de reproduction.

\section{Conclusion : Nous théorisons pour représenter et représentons pour intervenir}

Les constatations des conséquences sociales et environnementales de l'actuel modèle de développement capitaliste du secteur agricole pampéen, à l'image du soja, doivent suffire en elles-mêmes, pour promouvoir une discussion concernant la non-viabilité sociale du modèle et pour nous inviter à proposer de nouvelles alternatives qui ne soient pas au service des principes d'accumulation du capital, de l'efficacité productive ou de la spéculation financière. La science sociale ne doit pas se restreindre à décrire, expliquer et prévoir suivant les concepts de la science positive. Les scientifiques sociaux ont le devoir fondamental de dénoncer pour pouvoir transformer. Nous devons partir d'une approche critique du modèle afin de comprendre complètement son 
fonctionnement, détecter ses fissures et, comme le dit Mauricio Langón (2005), philosophe uruguayen, " souffler en elles ».

La théorisation nous permet de représenter mais la représentation est en même temps la base de l'intervention. Les catégories d'analyse exposées dans l'introduction (espace géographique et territoire) tentent d'exprimer une approche théorique, conceptuelle et méthodologique au problème posé. Mais elles doivent nécessairement être envisagées à partir d'un plan multidimensionnel idéologique et éthique, afin de pouvoir définir clairement : d'où ? Comment ? Dans quel but ? Pour qui ? Et avec qui ? Le territoire est le produit d'interrelations, de la potentialité de multiplicités et d'un permanent « être en train d'être ». Le tableau 2 expose le schéma de l'approche théorique - conceptuelle et métodologique - proposée. "Selon ce modèle de pensée, nous sommes conduits à déceler les liens établis, ceux qui sont en train de s'établir, ceux qui peuvent s'établir depuis le pouvoir hégémonique et ceux qui doivent s'établir à partir de la proposition d'un espace pour une analyse éthique et politique selon une perspective de responsabilité sociale et de contribution au bien public, tout en pensant le territoire comme patrimoine social commun » (Maldonado, Cóccaro, 2006, p. 28).

Tableau 2 : Légitimation des représentations et relations de pouvoir Relations of power and legitimisation of representations

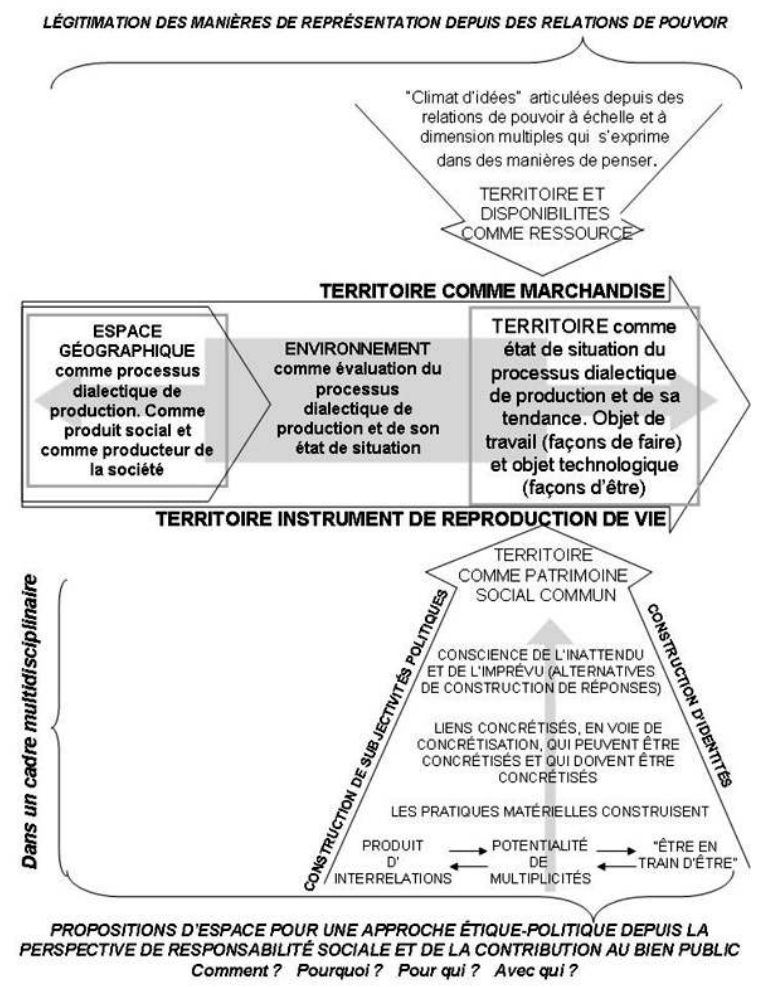

Le soja surprend la vulnérabilité sociale du contenu du territoire. Il surprend la vulnérabilité réelle et structurelle qui reflète les inégalités " nécessaires et fonctionnelles » produites par le système. Aujourd'hui, le prétexte est le soja, comme une façon de penser le territoire. 


\section{BIBLIOGRAPHIE}

AGÜERO (R.), GONZÁLEZ (J.), PUIGDOMENECH (E.), 2004. - « Diagnosis, problemáticas y perspectivas de la localización de la PEA Agropecuaria. La región pampeana que rodea a la ciudad de Río Cuarto », Revista Reflexiones Geográficas, n 11, p. 249-275.

AzCUY AMEGHino (E.), 2004. - « De cultivo marginal a motor de la agriculturización : hipótesis y problemas en torno a la "sojización" ", Dossiers du CIEA. n², p. 65-75.

AzcuY AMEghino (E.), 2007. - « "Pruebe a nombrar de memoria cinco empresas que estén explotando..." Propiedad y renta de la tierra en Argentina a comienzos del siglo XXI », Revista Interdisciplinaria de Estudios Agrarios, $\mathrm{n}^{\circ} 26$ y 27, p. 123-140.

CENTRo de estudios Para el desarRollo argentino, 2008. - « El trabajo en la Argentina », dossier $n^{\circ}$ 15, Buenos Aires, Argentine, $19 \mathrm{p}$.

Cisneros (J.), DE PRADA (J.), Degionani (A.), CANTERo GUtierRez (A.), GiL (H.), REYNero (M.), SHAH (F.), BRAVo URETA (B.), 2004. - « Erosión hídrica y cambios de uso de los suelos en Córdoba : Evaluación mediante el modelo RUSLE 2 », Actas del XIX Congreso Argentino de la Ciencia del Suelo, Paraná, Entre Ríos, Argentina, CD-ROM, 13 p.

Cisneros (J.), DE PRADA (J.), DEGionani (A.), CANTERo GUTIERREZ (A.), GIL (H.), REYNERo (M.), SHAH (F.), BRAVO URETA (B.), 2005. - « Potencial de escurrimiento de cuencas agrícolas en relación a los cambios de uso entre 1986 y 1999 », CONAGUA 2005, XX Congreso Nacional del Agua. Dpto. Gral de Irrigación, Mendoza, Argentina, CD-ROM, 12 p.

ChOlAKy (C.), REYNERo (M.), GONZÁLEZ (J.), CiSNEROS (J.), DEGIONANi (A.), CANTERo, GUTIERREZ (A.), 2005. - « Avance de la erosión lineal y producción de sedimentos en los arroyos menores del sur de Córdoba », CONAGUA 2005, XX Congreso Nacional del Agua. Dpto. Gral de Irrigación, Mendoza, Argentina, CD-ROM, 15 p.

Cóccaro (J.), VALENZuela (M.), SOSA (E.), GÓMEZ (O.), BOETTO (A.), SÁNCHEZ (L.), 1998-2002. - « El proceso de organización espacial del sur cordobés un estudio de base para desentrañar sus problemas ambientales. » Proyecto de investigación, Secretaría de Ciencia y Técnica de la Universidad Nacional de Río Cuarto, Córdoba, Argentina, informe final, 56 p.

CócCARO (J.), VALENZUELA (M.), SOSA (E.), GÓMEZ (O.), 2006. - « Expansion du soja et problèmes environnementaux, au sud de la province de Córdoba (Argentine) ", La Géographie, n 1521, p. 15-37.

CUfRÉ (D.), 2008. - « Los síntomas del bienestar. », Journal Página 12, 6 avril 2008,p. 2.

Journal Clarín, 2006. - « Casi el 70 \% de los cultivos se trabaja con siembra directa », rubrique $E l$ País, 24 août 2006.

JouRnal CLARÍn, 2007. - « Estiman una cosecha récord de 85 millones de toneladas », rubrique El País, 11 janvier 2007.

JOURNAL LA NACIÓN. - « La agricultura familiar produce más del 50 \% del empleo rural », rubrique Campo, 24 mars 2007.

JouRnal la naCión. - « La Propiedad de la Tierra », Editorial, 4 novembre 2006.

Di MÉo (G.), 1998. - Géographie sociale et territoires, Éditions Nathan, Paris, 320 p. 
FiLGUeIRA (C.), 2006. - « Vulnerabilidad social y oportunidades. », dans Política y gestión, Revista de la Escuela Política y Gobierno (Universidad Nacional de San Martín), Facultad de Derecho y Ciencias Sociales (Universidad Nacional del Nordeste), Centro de Estudios Interdisciplinarios (Universidad nacional de Rosario), Centro de Estudios Históricos (Universidad Nacional del Litoral), centro de Análisis Políticas Públicas (Universidad de Chile), CIESU (centro de Investigaciones y estudios Sociales del Uruguay), Colegio de Sociólogos de Uruguay, Homo sapiens Ediciones, Rosario, Argentina, p. 19-64.

FRIGERIO (R.), 1953. - Introducción al estudio del problema agrario argentino, Ed. Clase Obrera, Buenos Aires, $168 \mathrm{p}$.

GiberTi (H.), 1998. - « Tipos de cambios fundarios », Cuadernos del PIEA, nº 6, p. 61-69.

GómEz (R.), 2003. - Neoliberalismo Globalizado. Refutación y debacle, Ediciones Macchi, Buenos Aires, $200 \mathrm{p}$.

Grobocopatel (G.), 2005. - « Hemos aprendido a pescar », Suplemento Clarín Rural, 20 août 2005.

HARVEY (D.), 1998. - La condición de la posmodernidad. Investigación sobre los orígenes del cambio cultural, Amorrortu, Buenos Aires, $401 \mathrm{p}$.

HARVEY (D.), 2004. - El nuevo imperialismo, Ediciones Akal, Madrid, 178 p.

-, 2007. - Espacios del capital. Hacia una geografía crítica, Ediciones Akal,Madrid, 448 p.

HUERGo (H.), 2006. - « Cortar la onda “anti-soja” », suplemento Clarín Rural, 21 octobre 2006.

LANGóN (M.) 2005. - « Reflexiones para una educación filosófica en tiempos de globalización », dans Bernales Alvarado (M.), Lobosco (M.) (comp.), Filosofía, educación y sociedad global, Buenos Aires, Ediciones del Signo, p. 69-83.

LeFEBVRE (H.), 1984. - La producción del espacio, Ed. Anthropos, Barcelona, 485 p.

Maldonado (G.), Cóccaro (J.), 2006. - « Pensar el riesgo desde una aproximación geográfica. Lecturas de la problemática ambiental del sur cordobés », Revista Reflexiones Geográficas, $\mathrm{n}^{\circ}$ 12, p. 18-29.

MALDONADo (G.), 2008. - « Riesgo, agua y conflictos territoriales. Inundaciones del sur de la provincia de Córdoba, Desde 1970 a la actualidad », Plan de tesis doctoral, Universidad de Buenos Aires, $38 \mathrm{p}$.

Marx (K), 2001. El Capital. Crítica de la Economía Política. Fondo de Cultura Económica, 1946 México (Première édition en allemand 1867), tres tomos, 2425 p.

MASSEY (D), 2005. - « La filosofía y la política de la espacialidad: algunas consideraciones », dans Arfuch (L.) (comp.), Pensar este tiempo. Espacios, afectos, pertenencias, Buenos Aires, Paidós, p. 103-127.

PLAN FÉNIX, 2003-2008, Proyecto Estratégico de la Universidad de Buenos Aires, Journal Página 12, 27 mars, p. 10.

Pengue (W.), 2003. - «El vaciamiento de las pampas », Le Monde Diplomatique, $\mathrm{n}^{\circ} 47$.

-, 2005. - Agricultura industrial y transnacionalización en América Latina. ¿La transgénesis de un continente?, GEPAMA y Programa de Naciones Unidas para el Medio Ambiente, México, 221 p.

Pucciarelli (A), 2008. « No hay propuestas detrás de la puja », Journal Página 12, 2 juin 2008, p. 8.

RAPOPORT (M.), 2007. - Historia de la economía argentina del siglo XX, Fascículo 1, Ed. La Página, Buenos Aires, Argentina, 14 p. 
RoBIN (M.), 2008. - El mundo según Monsanto. De la dioxina a los OGM. Una multinacional que les desea lo mejor, Ediciones Península, Madrid, 528 p.

RodrigueZ (C.), 2008. - «Cercados por la soja », Journal Página 12, 28 septembre 2008, p. 22.

Rofman (A.), 2008. « Las economías regionales. El otro campo », suplementoEconómico Cash, Página 12,20 juillet 2005, p. 4.

SANTos (M.), 1985. - Espaço \& metodo, Sao Pablo, Nobel, 88 p.

-, 1994. - « O retorno do territorio », dans Santos (M.) et al.,Territorio, globalizaçao e fragmentaçao, Sao Pablo, p. 15-20.

-, 1996.- Metamorfosis del espacio habitado. Barcelona, Oikos-tau. 118 p.

-, 2000. - La naturaleza del espacio. Técnica y tiempo. Razón y emoción, Ariel, Barcelona, 348 p.

-, 2002. - El presente como espacio. Biblioteca básica de geografía, Sistema Universidad Abierta,

Facultad de Filosofía y Letras, Universidad Autónoma de México, México, serie traducciones, 38 p.

SEMINARIO (E.), 2001. - « “Chacrings" al ataque », Suplemento Clarín Rural, 8 septembre 2001.

SHIVA (V.), 2003. - Cosecha robada. El secuestro del suministro mundial de alimentos, Buenos Aires, Paidós SAICF, $166 \mathrm{p}$.

SILVEIRA (M.), 1999. - « Imperio de la escala, escala del Imperio », Revista Universitaria de Geografía, Universidad Nacional del Sur, vol. 8, n 1 y 2, p. 105-113.

-, 2001. - « Una situación geográfica : desde la teoría a la historia, de la historia a la teoría », Estudios Socioterritoriales. Revista de Geografía, n² 2, p. 157-167.

-, 2003. - « Por una epistemología geográfica », Dans Bertoncello (R.), Alesandri (C.) (comp.), Procesos Territoriales en Argentina y Brasil, Instituto de Geografía, facultad de Filosofía y Letras, Universidad de Buenos Aires, p. 13-26.

-, 2008. - «Los territorios corporativos de la globalización », dans Geograficando, Revista de Estudios Geográficos, Departamento de Geografía, Facultad de Humanidades y Ciencias de la Educación, Universidad Nacional de La Plata, p. 13-26.

Sosa (E.), VAlenzuela (M.), cóccaro (J.), MALDonado (G.), GóMEZ (O.), BOETTO (A.), SÁNCHeZ (L.), 2005-2006. - « Problemáticas territoriales y medio ambiente: riesgos emergentes en el sur de Córdoba. Lecturas situadas que interpelan responsabilidades », Proyecto de Investigación, Secretaría de Ciencia y Técnica de la Universidad Nacional de Río Cuarto, informe final, 48 p.

TEUbAl (M.), giACARRA (N.), 2005. - « Transformaciones agrarias en la Argentina. Agricultura industrial y sistema agroalimentario. "Dans GIACARRA (N.), TEUBAL (M.) (coord.), El campo argentino en la encrucijada. Estrategias y resistencias sociales, ecos en la ciudad, Alianza editorial, Buenos Aires, p. 37-78.

Teubal (M.), 2006. - « Expansión del modelo sojero en la Argentina. De la producción de alimentos a los commodities », dans Realidad Económica, n²20, publication du IADE (Instituto Argentino de la Empresa), mai-juin 2006, p. 71-96.

TRUcco (V.), 2000. - Oportunidades y Desafíos de la Biotecnología para la Agricultura del Mercosur, AAPRESID, DRCLAS, CD-ROM.

-, 2004. - « Es cuestión de darse cuenta », suplemento Clarín Rural, 2 octobre 2004.

-, 2003. - «El desafío de darse cuenta », suplemento Clarín Rural, 6 septembre 2003. 
VALENZUELA (M.), SOSA (E.), CócCARO (J.), GÓMEZ (O.), MALDONADO (G.), SÁNCHEZ (L.), BOETTO (A.), 2003-2004 . - « La gestión de riesgo en el corredor central del Mercosur, sector Chaján - Villa María. Un instrumento para el desarrollo regional. »Proyecto de Investigación, Secretaría de Ciencia y Técnica de la Universidad Nacional de Río Cuarto, Córdoba, Argentina, informe final, 53 p.

ZAIAT (A.), 2008. - « El arrendatario », Suplemento Económico Cash n946, Journal Página 12, 18 mai 2008 , p. 8.

\section{NOTES}

1. L'Argentine se divise en 23 provinces, divisions juridiques, politiques et administratives, équivalentes des départements français.

2. Région de la province de Córdoba, au Sud du Río Tercero.

3. C'est la dénomination que les auteurs ont adoptée pour faire référence aux indigènes ou aborigènes qui habitaient le territoire argentin et le continent américain avant la colonisation.

4. Le début de l'accumulation a requis l'élimination totale des peuples originaires au Sud de la région pampéenne et en Patagonie. Celle-ci a été réalisée par le biais de campagnes militaires terminées en 1879.

5. Système qui permet de louer la terre à ses propriétaires contre de l'argent ou une partie de leur récolte.

6. Système de travail rural où le revenu est partagé entre le « aparcero » (celui qui travaille la terre) et le propriétaire.

7. Strate herbacée ayant des espèces qui poussent en hiver et d'autres en été. De nos jours, ce biome a un haut degré d'artificialisation à cause des activités d'agriculture et d'élevage. La quasitotalité des communautés végétales originelles ont disparu.

8. Formation arborée et arbustive épineuse dans des régions arides et semi-arides.

9. Désigne une économie qui vient s'appuyer à nouveau sur le secteur primaire.

10. Modèle d'utilisation agricole du sol basé sur une forte croissance de l'agriculture dans les agro-systèmes. Il se caractérise par la réalisation de pratiques intenses de labourage comme conséquence de la modernisation productive.

11. Expansion de la culture du soja, avec des caractéristiques de monoculture.

12. Selon la définition de Walter Pengue (2005), c'est le processus d'exportation d'activités propres à la Pampa, vers des régions extra-pampéennes.

13. Expression désignant l'espace où différentes activités rurales sont dominantes. Ce concept implique une grande diversité d'activités et d'échelles de travail.

14. Expression que les auteurs utilisent pour signaler que, derrière les « avantages » proclamés par les tenants de la "sojisation ", se cachent des impacts sociaux et environnementaux négatifs.

15. La dévaluation a eu lieu en 2001, où il est établi une parité entre le dollar et le peso argentin de 1 pour 3.

16. Le secteur central est la surface la plus productive et rentable de la région pampéenne. Cette région comprend les provinces de Buenos Aires, La Pampa, Santa Fe, Córdoba et Entre Ríos, et constitue $40 \%$ des 175 millions d'hectares de la superficie utilisée dans le pays (Recensement national agricole 2002).

17. Selon l'avis des auteurs, ce phénomène peut expliquer l'union circonstantielle de tous ceux qui dirigent les affaires rurales, c'est-à-dire, les diverses entités rurales qui sont entrées en conflit avec le gouvernement au premier trimestre de 2008, sur la question des rétentions du soja (droits d'exportation). La Société Rurale Argentine (SRL) et les Confédérations Rurales Argentines (CRA) qui, tout au long de l'histoire, ont protégé les intérêts des propriétaires fonciers, sont devenues conjointement avec la Fédération Agricole Argentine (FAA) et les Confédérations 
intercoopératives agricoles (Coninagro), les représentantes des petits et moyens producteurs des terres pampéanisées. En revanche, le petit producteur des économies régionales qui exploite 5, 10, ou 20 hectares, n'est pas protégé par ses organismes.

18. Nom qui désigne le mouvement de protestation des entrepreneurs et des producteurs, maîtres des moyens de production, afin de la différencier de la grève des salariés. Le lockout manifestait contre les retentions mobiles (droits d'exportation) sur les prix du soja, établis par le gouvernement argentin en mars 2008.

19. Les pools de semailles apparaissent depuis les années 1980 et se développent intensément pendant les années 1990 conjointement avec l'avancée de la sojisation. Tout au début, ils étaient portés par les producteurs qui devaient augmenter l'échelle de leur production afin de se maintenir économiquement. Mais avec le boom de l'agro-business, ils sont devenus une alternative attrayante pour les investisseurs. Il s'agit d'une sorte d'organisation dont les capitaux sont spéculatifs. Son fonctionnement consiste à affermer des unités d'exploitation et à négocier avec les entrepreneurs des machines agricoles afin de produire, pendant une période de temps déterminée, avec un important investissement. Selon un rapport de l'Associationdeconsortia régionaux d'expérimentation(Aacrea), à travers les consortiums régionaux d'expérimentation agricole (CREA), on estimait que la superficie contrôlée par les pools atteignait les 2 millions d'hectares en 2002. Il existe des différences entre les pools (qui sont des fonds d'investissement) et les grandes entreprises agricoles (céréales et bétail). Par exemple, l'entreprise Los Grobo (famille Grobopocatel) possède plus de 17700 hectares, mais elle cultive un total de plus de 150000 hectares, produit 1,5 million de tonnes mais commercialise plus de 112000 tonnes de farine. Dans l'ensemble, les entreprises du holding facturent quelque 200 millions de dollars par an. Los Grobo est aussi présente en Uruguay, au Paraguay et au Brésil et cherche à semer un total de 400000 hectares dans le Mercosur. Deux autres entreprises importantes sont Adecoagro (Georges Soros) et El Tejar. La première possède 225000 hectares en Argentine, en Uruguay et au Brésil et la deuxième, plus de 150000 hectares dans ces trois pays. Également, il existe en Argentine une liste des quinze plus grandes entreprises et pools de semailles, établie par Neil P. Richardsson, de l'Université de Californie (Teubal, $2005 ; 2006$ ).

20. Les " propriétaires qui prennent des terres " : expression qui sert à désigner - d'après le Recensement national agricole de 2002- les propriétaires qui, en plus d'exploiter leurs terres, cultivent d'autres terres affermées ou prises à bail. Ils représentent $36 \%$ (24,5 millions d'hectares) de la terre cultivée dans la région contre 50,5\% (34,5 millions d'hectares) des propriétaires qui exploitent seulement leurs terres. Entre le Recensement national agricole de 1988 et celui de 2002, ces deux catégories ont augmenté de moitié. Mais si on tient compte de la superficie totale, la terre additionnelle que les propriétaires ont prise à bail a triplé l'accroissement de la superficie des investisseurs purs (5,8 millions d'hectares contre 1,9 millions d'hectares). Les pools de semailles sont inclus dans cette même catégorie.

21. Censo Nacional Agropecuario, Instituto Nacional de Estadística y Censos. Ministerio de Economía de la Nación, Buenos Aires, Argentina, 1988 et 2002.

22. Ces entreprises se situent parmi les dix exportatrices les plus importantes du pays. En 2007 elles ont exporté pour un total de 14 milliards de dollars, ce qui représentait $26,6 \%$ du total des exportations argentines.

23. Pendant les premières années des privatisations, le transport de charge par voie ferrée était considéré comme une affaire non rentable pour les adjudicataires. Aujourd'hui, il est devenu une exploitation hautement rentable grâce aux volumes des récoltes et aux exportations, et grâce aux conditions avantageuses qui ont été stipulées dans les contrats signés. L'entreprise Ferroexpreso Pampeano, ligne opérée par Techint, dessert la région céréalière entre Rosario et Bahía Blanca. $80 \%$ de son revenu provient du transport des grains. Pendant les deux premiers mois de 2008, elle a augmenté son activité de 89,2\%. D’autre part, le Nuevo Central Argentino, ligne contrôlée 
par Aceitera General Deheza (Huilerie Générale Deheza) - famille Urquía -, dans la province de Córdoba, relie la ville de Córdoba au port de Rosario (province de Santa Fe). Pendant les deux premiers mois de 2008 , le nombre de voyages a augmenté de $36,8 \%$ par rapport à la même période de 2007. Presque la moitié de cette augmentation correspond aux oléagineux dont le soja qui est le plus important. Alors que sa consommation intérieure est la moins importante (Instituto Nacional de Estadística y Censos, primer bimestre 2008).

24. Les agrochimiques sont les insecticides, fongicides et herbicides, employés pour combattre les ravageurs.

25. On sème directement le sol, en introduisant les grains dans une coupure verticale, ayant peu de centimètres de profondeur, qui se réalise avec une lame circulaire sans labourer la terre. Cette technique exige le contrôle préalable des mauvaises herbes avec des herbicides et la fertilisation, car la minéralisation naturelle des nutriments du sol se produit très lentement. Ce type de labour est appuyé par AAPRESID (Association argentine des producteurs de semis direct), agent actif dans la consolidation du paquet technologique.

26. L'autorisation d'introduire la culture du soja RR a eu lieu en 1996 par une régulation administrative provenant du secrétariat de l'Agriculture, Élevage, Pêche et Aquaculture, sans débat public ni participation du Congreso de la Nación (Assemblée nationale) sous la présidence de Carlos Menem.

27. DuPont-Pioner (États-Unis), Monsanto (États-Unis), Novartis (Suisse), Groupe Limagrain (France), Advanta (Royaume-Uni et Hollande), Guipo Pulsar-Seminis-ELM (Mexique), Sakata (Japon), KWSHG (Allemagne), Y Taki (Japon) (Shiva, 2003).

28. Gómez ne fait pas référence au processus de sojisation, mais interprète le néolibéralisme depuis ses propres présupposés pour en établir, dans ce cadre, une critique de réfutation.

29. Comité national pour les grains et Comité national pour la viande. Ils ont été désarticulés pendant le gouvernement de Carlos Menem (1989-1999). Encore une preuve de l'application approfondie du modèle néolibéral. Ces Comités avaient été créés pendant les gouvernements conservateurs (1932) postérieurs à la crise mondiale des années 1930 pour faire face à l'effondrement des exportations agricoles. Les Comités achetaient leur production aux producteurs à des prix plus élevés que les prix internationaux prenant à leur charge les pertes (commentaire des auteurs).

30. Institut national de technologie pour l'agriculture et l'élevage, fondé en 1956 sur la base de départements techniques et de stations expérimentales qui existaient au Ministère de l'Agriculture et de l'Élevage de l'époque. Même s'il est autonome administrativement et autarcique, il dépend fonctionnellement du Secrétariat de l'agriculture, de l'élevage, de la pêche et de l'alimentation de la Nation.

31. Les départements sont des divisions juridiques, politiques et administratives des provinces de l'Argentine. Seule la Province de Buenos Aires est divisée en " partidos » ayant la même entité que les départements.

32. Ceci n'a pas impliqué le déplacement de l'élevage dans des terres marginales. Les pâturages pérennes semés se sont étendus par opposition aux champs naturels. Ceci montre une augmentation de l'élevage mais aussi sa concentration dans des superficies plus réduites, un pâturage plus intensif dans des prairies pérennes cultivées et de culture annuelle. Les feedlots : engraissement de bétail dans des parcs avec des aliments composés.

33. Certaines provinces du NEA (Nord-Est Argentin) telles que Formosa ou Chaco sont particulièrement affectées. Dans ces provinces, la superficie cotonnière a souffert d'un important déplacement à cause de l'expansion du soja. La production de coton est liée à l'identité sociale et culturelle de la région. De petits producteurs ayant cinq à dix hectares sont obligés de vendre leurs terres à de gros producteurs venant des provinces de Buenos Aires, Córdoba ou Santa Fe pour produire du soja. La province du Chaco a une superficie cultivable de 1,5 million d'hectares. Le Recensement national agricole 2002 a déclaré 220000 hectares semés de soja, tandis qu'un 
relèvement par satellite de la province réalisé pour la campagne agricole de 2008 a déclaré 684 000 hectares. Inversement, les cultures de coton qui occupaient 712000 hectares en 1997-1998, se sont réduites à 180000 hectares en 2008. La valeur de l'hectare de terre y est passée de 50 pesos en 2002 à 8000 pesos en 2008 ! La valeur moyenne de la parité du change étant, depuis 2001, de 1 dollar pour 3 pesos argentins.

34. Le secrétariat de la Politique environnementale de la province de Salta a convoqué à 211 audiences publiques entre 2004 et 2007 parmi lesquelles 191 l'ont été pour cause de défrichement. On est passé de 129257 ha dévastées en 2004, à 435399 en 2007. Cette tendance est en augmentation. L'Université nationale de Salta réclame la suspension des activités de défrichement qui avaient été autorisées dans la région du Chaco Salteño avant l'entrée en vigueur de la Loi des Bois promulguée au mois de novembre de 2007 par le Congreso Nacional (Assemblée nationale). Cet arrêt doit être appliqué pour garantir la survie des peuples originaires et pour faire face à la menace de la continuité biologique et culturelle (Rodriguez, 2008).

35. Anglicisme de l'auteur pour faire référence au " chacarero » (fermier), expression utilisée dans la région pampéenne pour désigner l'exploitation familiale, généralement dans de petites unités de production.

36. L'étude et les programmes se trouvent en ligne dans la page web de la ONG « Responde » [www.responde.org.ar].

37. Propos de Jorge Iglesias, date inconnue.

38. Une étude réalisée entre 2004 et 2007 par une équipe de recherche formée par des membres de l'Hôpital Italiano de Rosario, le Centre de recherche en biodiversité et environnement, l'Université Nationale de Rosario et l'Institut national de technologie agricole, affirme que certains produits agrochimiques peuvent perturber la physiologie hormonale des bébés, enfants, adolescents et adultes.

39. Quelques documents officiels et travaux de recherche effectués par la Faculté de Médecine de l'Université de Buenos Aires déconseillent leur consommation, notamment chez les enfants de moins de deux ans. Le soja ne contient pas d'acides aminés. Les problèmes de malnutrition en Argentine sont associés principalement au déficit de micronutriments (vitamines, fer, zinc, cuivre et calcium) plutôt qu'au manque de protéines. La haute concentration de phytates dans le soja nuit à l'absorption de ces micronutriments, notamment à celle du fer, qui évite les anémies et celle du zinc, si important dans les processus immunitaires.

40. Le 17 avril 2008 a été créé le Front National paysan à l'aide du Mo. Ca. Se (Santiago del Estero), MOCAFOR (Formosa), Hijos de Monte (Province de Salta), la COOPAFES (Coopérative des producteurs de l'agriculture familiale et de l'économie sociale) de la province de Mendoza, ONPIA (Organisation nationale des peuples indigènes en Argentine) et le Bureau d'organisation des producteurs de Buenos Aires.

41. Selon des données de la Direction de Bois du secrétariat de l'Environnement de la Nation, pendant la période 1998-2002, environ 920000 ha ont été déboisés. Actuellement, le déboisement des forêts autochtones est de 230000 ha par an, avec la circonstance aggravante de l'avancée sur des territoires habités par des familles avec une rationalité différente de celle qui est liée à cette expansion.

42. Lorsqu'il a fallu expliquer les causes des inondations de la ville de Santa Fe, produites par le débordement du fleuve Salado, au mois d'avril en 2003, après une période d'intenses précipitations, on n'a pas considéré l'impact du soja sur l'environnement. En effet, le défrichement du bassin du fleuve Salado en vue de l'ensemencement des terres avec du soja, n'a pas été considéré parmi les causes des inondations.

43. Il est intéressant de montrer les conditions du travail salarié dans le secteur de la culture des céréales, des oléagineux et des fourrages. Selon des données disponibles l'année 2006, 1265000 d'emplois existants, 62,5\% n'étaient pas déclarés, ce qui représente le taux d'informalité le plus haut de toute l'économie. Le salaire des travailleurs dans cette condition est 35,6\% inférieur au 
salaire que touche un travailleur déclaré (CENDA, 2008). Le statut de l'ouvrier agricole a été une politique emblématique du premier péronisme pendant la deuxième moitié des années 1940. En 1976, la dictature a qualifié les ouvriers agricoles comme les «cousins pauvres» des ouvriers salariés (car ils sont les plus défavorisés des ouvriers). Ce gouvernement a reformulé la loi de Contrat de Travail promulguée précédemment par le gouvernement péroniste, ce qui a représentéune avancée manifeste du secteur patronal. "Les travailleurs ruraux sont restés en dessous de leurs camarades de classe » annonce la loi de Travail sur la modification du régime de travail agricole envoyée au Congreso Nacional pour son traitement. Quelle sera la réaction des entités agricoles lorsqu'on avance sur un système plus adéquat pour le travailleur rural? (Commentaire des auteurs).

44. Horacio Giberti (1998) élabore un travail où il différencie conceptuellement évolution et réformes agraires selon leurs diverses variables : l'évolution peut être spontanée, induite et dirigée ; et les réformes peuvent comporter ou non des changements de système. Selon ces principes, l'Amérique Latine a généralement été l'acteur principal de réformes agraires sans changement de système.

45. Le sous-secrétariat de l'Agriculture Familiale et du Développement Rural a été créé en 2008. Il dépend du Secrétariat de l'Agriculture, de l'Élevage, de la Pêche et de l'Alimentation. Son travail atteint les petits producteurs ayant de petites unités d'exploitation consacrées principalement à la production d'aliments, avec un petit capital et ne faisant pas partie du schéma de commercialisation des chaînes agroalimentaires. Le Centre de Recherche et de Développement pour la Petite Agriculture Familiale (Cipaf) affirme que les exploitations agricoles familiales représentent plus de $66 \%$ dans le pays et génèrent $53 \%$ de l'emploi rural. (journal La Nación, 2007).

46. $50 \%$ de la consommation alimentaire dans les foyers argentins provient de l'agriculture familiale : le petit producteur de légumineux, légumes, fruits, herbe à maté, thé, herbes aromatiques, etc. C'est le soutien qui vise à éviter la migration de la population rurale des provinces argentines. C'est l'assurance de l'occupation des terres de la part des familles productrices. Ceci exige une attention permanente en termes de qualité de la vie et des biens publics qu'ils doivent recevoir : santé, éducation, logement garantissant leur permanence et non pas l'exode (Rofman, 2008).

47. On fait référence à la possession, contrôle et/ou mobilisation des ressources matérielles et symboliques permettant à chaque individu de se débrouiller dans la société (capital humain, expérience professionnelle, niveau éducatif, composition et attributs de la famille, capital social, participation à des réseaux, capital physique, etc).

48. La structure d'opportunités provient du marché, de l'État et de la société. Dans ce dernier cas, elle provient du domaine des institutions et des rapports sociaux.

\section{RÉSUMÉS}

Depuis la grande expansion productive de la région pampéenne argentine, initiée dans la seconde moitié du $\mathrm{XIX}^{\mathrm{e}}$ siècle, le pays s'est structuré autour d'une région centrale à forte productivité agricole (végétale et animale), entourée d'économies régionales destinées principalement à la consommation intérieure. 
À partir des années 1970, émerge le processus dit d'agriculturisation, accéléré au cours des quinze dernières années, caractérisé par l'importance croissante du rôle joué par le soja transgénique. Ce processus s'appuie sur la mise en place de plusieurs conditions structurales préalables, et sur l'irruption d'un modèle technologique qui a permis son développement optimal. À partir de l'articulation de ces conditions, la production de soja en Argentine s'est intensifiée et étendue, estompant les traditionnelles différences agro-écologiques régionales, homogénéisant le modèle de production à l'image du soja.

Cette "sojisation » a produit l'émergence de nouveaux acteurs sociaux et d'une nouvelle configuration du réseau traditionnel de production de la région pampéenne. Un modèle social inégalitaire se construit ainsi, sur la base d'inégalités antérieures toujours irrésolues. Il en résulte l'accentuation des différences sociales et l'émergence de différents degrés de vulnérabilité sociale et environnementale.

Ce processus met en évidence que la production de soja témoignage d'une façon de penser le territoire aujourd'hui, selon des principes néolibéraux qui le légitiment comme une simple marchandise. C'est face à cette situation que la nécessité de penser le territoire selon d'autres principes éthiques et politiques s'impose. Le défi est la proposition conceptuelle d'un espace pour une analyse éthique et politique, selon la perspective de la responsabilité sociale et de la contribution au bien public.

Since the beginning of the intensive developpement of Argentine pampean region, (1880's approximately), the whole country had build a new spatial structure, with Pampa as an agricultural core and small peripheral areas orientated toward national consumation.

From 1970 onward the agriculture activity has undergone a large shift towards agro busisness and transgenic soy has been expending. New conditions and new social actors were necessary to achieve this process. A new model has been set and the traditional distribution of regional economies is deseapearing. The national agricultural production is getting more and more homogenous.

This model of soy production has generated a social unequal structure that reinforce the previous social inequalities and the social and environmental vulnerability. Therefore, it is necessary to propose new ways to cope with inequality under another ethical-political assumption. Its base is to think the territory as an instrument of socio-territorial justice distribution.

Desde la gran expansión productiva de la región pampeana argentina, iniciada a mediados de la segunda mitad del siglo XIX, el país se configuró en torno a una región central fuertemente agrícolo-ganadera, rodeada de economías regionales orientadas, fundamentalmente, al consumo interno.

En la década de 1970 comienza el denominado proceso de "agriculturización", potenciado en los últimos quince años, con un fuerte protagonismo de la soja transgénica.

Dicho proceso ha necesitado del establecimiento de condiciones estructurales previas y la irrupción de un modelo tecnológico que permite su óptimo desarrollo. De la articulación de estas condiciones se intensifica y expande la producción sojera en el país. Se desdibujan las tradicionales diferencias agroecológicas regionales y se homogeniza el modelo de producción con el rostro de la soja.

Esta "sojización" ha generado la emergencia de actores sociales nuevos y una nueva configuración de la trama productiva tradicional de la región pampeana. Se construye un modelo social desigual asentado sobre desigualdades precedentes que no han sido resueltas. Se amplían así las diferencias sociales y se generan grados de vulnerabilidad social y ambiental.

Este proceso pone en evidencia que la producción de soja es testimonio de una forma de pensar el territorio hoy, bajo supuestos neoliberales. Así, se legitima el territorio como una mera mercancía y plantea la necesidad de pensarlo bajo otros supuestos ético-políticos. El desafío es la 
proposición conceptual de un espacio para un abordaje ético- político desde la perspectiva de responsabilidad social y de aporte al bien público.

\section{INDEX}

Mots-clés : agriculture, Soja, territoire, patrimoine

Palabras claves : Soja, territorio patrimonio social común, territorio-mercancía, vulnerabilidad socio-ambiental

Index géographique : Argentine

Keywords : soy, heritage, agriculture, territory

\section{AUTEURS}

\section{JOSÉ MARÍA CÓCCARO}

Département de Géographie, Faculté de Sciences Humaines, Université nationale de Río Cuarto, Ruta Nacional no 36, km 601, Río Cuarto - CP 5800 Córdoba, Argentine,

jmcoccaro@yahoo.com.ar

\section{GABRIELA INÉS MALDONADO}

Département de Géographie, Faculté de Sciences Humaines, Université nationale de Río Cuarto, Ruta Nacional nº 36, km 601, Río Cuarto - CP 5800, Córdoba, Argentine, gimaldonado@hum.unrc.edu.ar 